\title{
1 Parallel genetic changes underlie integrated craniofacial traits in an adaptive radiation of
}

\section{2 trophic specialist pupfishes}

4 Michelle E. St. John ${ }^{1,2}$, Julia C. Dunker ${ }^{1}$, Emilie J. Richards ${ }^{1,2}$, Stephanie Romero ${ }^{3}$, Christopher

5 H. Martin ${ }^{1,2}$

$7 \quad{ }^{1}$ Department of Integrative Biology, University of California, Berkeley, CA 94720

$8 \quad{ }^{2}$ Museum of Vertebrate Zoology, University of California, Berkeley, CA 94720

$9{ }^{3}$ Department of Evolution and Ecology, University of California, Davis, CA 95616

12 Keywords: quantitative trait loci, ecological speciation, functional morphology, ecomorph,

13 lepidophagy, durophagy

14 Correspondence: chmartin@berkeley.edu 


\section{Abstract}

17 Many factors such as divergence time, shared standing genetic variation, frequency of

18 introgression, and mutation rates can influence the likelihood of whether populations adapt to

19 similar environments via parallel or non-parallel genetic changes. However, the frequency of

20 parallel vs non-parallel genetic changes resulting in parallel phenotypic evolution is still

21 unknown. In this study, we used a QTL mapping approach to investigate the genetic basis of

22 highly divergent craniofacial traits between scale- and snail-eating trophic specialist species

23 across similar hypersaline lake environments in an adaptive radiation of pupfishes endemic to

24 San Salvador Island, Bahamas. We raised F2 intercrosses of scale- and snail-eaters from two

25 different lake populations of sympatric specialists, estimated linkage maps, scanned for

26 significant QTL for 30 skeletal and craniofacial traits, and compared the location of QTL

27 between lakes to quantify parallel and non-parallel genetic changes. We found strong support for

28 parallel genetic changes in both lakes for five traits in which we detected a significant QTL in at

29 least one lake. However, many of these shared QTL affected different, but highly correlated

30 craniofacial traits in each lake, suggesting that pleiotropy and trait integration should not be

31 neglected when estimating rates of parallel evolution. We further observed a $23-52 \%$ increase in

32 adaptive introgression within shared QTL, suggesting that introgression may be important for

33 parallel evolution. Overall, our results suggest that the same genomic regions contribute to

34 parallel integrated craniofacial phenotypes across lakes. We also highlight the need for more

35 expansive searches for shared QTL when testing for parallel evolution. 


\section{Introduction}

40 Convergent evolution describes the independent evolution of similar phenotypes in response to

41 similar selective pressures and provides strong support for ecological adaptation (Losos 2009;

42 Schluter 2000). This includes both non-parallel genetic changes, such as the evolution of

43 antifreeze glycoproteins in icefishes or the 'thunniform' body shape of lamnid sharks and tunas

44 (Chen et al. 1997; Donley et al. 2004), and parallel genetic changes such as tetrodotoxin

45 resistance in snakes and pufferfishes or the evolution of voltage-gated sodium channels in

46 mormyrid and gymnotiform electric fishes (Hopkins 1995; Katz 2006; Jost et al. 2008; Feldman

47 et al. 2009; Zakon et al. 2006). Instances of convergence across independent lineages (i.e., across

48 groups that lack a recent common ancestor and shared genetic backgrounds) provide the

49 strongest evidence for adaptation; however, repeated evolution of similar phenotypes in response

50 to similar selective pressures among lineages derived from the same ancestral population can

51 also provide insight into the process of adaptation. Understanding this process, traditionally

52 known as parallel evolution (Futuyman 1986), is important because it can help to tease apart the

53 contributions of natural selection and shared genetic constraints to similar phenotypes (Schluter

54 et al. 2004; Stuart et al. 2017). Parallel phenotypic evolution can also occur via parallel or non-

55 parallel genetic changes (e.g., Cresko et al. 2004), but even non-parallel genetic changes

56 occurring in the same ancestral genetic background (e.g. Chan et al. 2010; Xie et al. 2019)

57 provide weaker evidence for adaptation than convergence across independent lineages due this

58 shared history. Despite substantial attention, the frequency and likelihood of parallel phenotypic

59 evolution via parallel or non-parallel genetic changes is still relatively unknown (Stern and

60 Orgogozo 2008; Stern 2013; Rosenblum et al. 2014). 
62 produced by parallel or non-parallel genetic mechanisms. First, recently diverged species exhibit

63 increased probabilities of genetic parallelism when adapting to similar environments. Recently

64 diverged taxa may inhabit similar environments more frequently or they may have similar

65 genetic architectures, similar genetic variance-covariance matrices, or similar genetic

66 backgrounds that produce similar epistatic interactions (Conte et al. 2012; Rosenblum et al.

67 2014). Second, any mechanism that allows the use of the same adaptive genetic mechanism

68 should increase the likelihood of convergence via parallelism, including the availability of shared

69 standing genetic variation and introgression (Rosenblum et al. 2014). For example, threespine

70 sticklebacks colonized freshwater thousands of times and converged on similar phenotypes

71 largely due to selection on an ancient shared pool of marine standing genetic variation (Jones et

72 al. 2012; Feulner et al. 2013; Nelson and Cresko 2018; Haenel et al. 2019; but see: Chan et al.

73 2010; Stuart et al. 2017). Similarly, increased adaptive introgression should also make genetic

74 parallelism more likely (Grant et al. 2004; Morjan and Rieseberg 2004; Hedrick 2013; Taylor et

75 al. 2020). Third, adaptive genetic variation with larger effect sizes and fewer pleiotropic effects

76 should be reused more frequently across populations, particularly when a population is far from a

77 new adaptive optimum (Linnen et al., 2013; Orr, 2005; Stern, 2013). Finally, de novo mutations,

78 large mutational target sizes, and polygenic adaptive phenotypes are more likely to result in

79 parallel phenotypic evolution via non-parallel genetic pathways (Wittkopp et al. 2003; Kowalko

80 et al. 2013; Bolnick et al. 2018, but see: Colosimo et al. 2004; Chan et al. 2010; Xie et al. 2019).

81 Quantitative trait locus (QTL) mapping is often used to infer whether parallel or non-

82 parallel genetic changes underlie parallel phenotypes. However, many QTL studies only

83 investigate a limited number of traits that are controlled by large effect loci, which may bias the 
84 literature towards supporting genetic parallelism (Conte et al. 2012). This bias may be

85 exacerbated by the fact that in many QTL studies the genomic regions associated with a parallel

86 phenotype are large, contain many genes, and their effects on phenotypic variance are

87 overestimated in under-powered studies (Beavis 1998). These methodological and experimental

88 limitations reduce confidence in the specific genomic regions associated with a parallel

89 phenotype and, by extension, reduce confidence in whether parallel evolution was due to parallel

90 or non-parallel genetic changes. One possible solution is to compare the genomic regions

91 associated with many different phenotypes across populations (Erickson et al. 2016). In this

92 scenario, shared genomic regions across populations provide strong support for genetic

93 parallelism, except in the likely rare instances of independent de novo mutations within the same

94 region (O’Brown et al. 2015; Xie et al. 2019; Chan et al. 2010).

95 The San Salvador Island (SSI), Bahamas pupfish radiation is an excellent system for

96 investigating the genetic underpinnings of parallel ecomorph phenotypes because novel trophic

97 specialists occur in sympatry across multiple hypersaline lake populations on the island. The

98 radiation includes three pupfish species: a generalist pupfish (Cyprinodon variegatus), a scale-

99 eating (lepidophagous) pupfish (C. desquamator), and a snail-eating (durophagous) pupfish $(C$.

100 brontotheroides; Martin and Wainwright 2013). The snail- and scale-eating pupfishes are

101 endemic to SSI and occur in sympatry with one another and the generalist pupfish.

Among lakes, specialists have converged on multivariate phenotypes that are adaptive for

103 their given ecological niche. For example, scale-eaters across all lakes exhibit increased oral jaw

104 size (Martin \& Wainwright, 2013; Hernandez et al. 2018) and reduced lower jaw angles during

105 scale-eating strikes which may play a critical role in scale-biting performance during high-speed

106 strikes on their prey (St. John et al. 2020b). Similarly, the snail-eating pupfish exhibits a novel 
nasal protrusion which may improve oral snail-shelling performance or result from sexual

108

109

110

selection (Martin and Wainwright 2013; St. John et al. 2020a). Furthermore, the nasal protrusion of the snail-eating species varies substantially among lake populations (Martin and Feinstein 2014; Hernandez et al. 2018). Despite the importance of these species characteristics, we still do not understand how their genetic architecture varies across populations.

There is some evidence to suggest that parallel genetic changes underlie specialist phenotypes on SSI. First, the SSI radiation is very young, diverging only about 10 kya (Hagey and Mylroie 1995). Second, previous genomic analyses show that many of the alleles associated with trophic specialization arrived on SSI from Caribbean-wide standing genetic variation within generalist pupfish populations, but there are also some de novo adaptive mutations associated with scale-eating (Richards et al. 2021). Scale-eaters form a monophyletic group, suggesting a shared genetic component to the scale-eating phenotype across lakes (Richards and Martin 2017). In contrast, snail-eaters and generalists often genetically cluster together by lake instead of by species_-suggesting that non-parallel genetic changes could underlie parallel snail-eater phenotypes across lakes (Martin and Feinstein 2014; Richards and Martin 2017). Furthermore, previous studies have documented strong genetic divergence between scale-eaters from Crescent Pond and all other populations of scale-eater (Richards \& Martin, 2017; Richards et al., 2021).

In this study we mapped the genetic basis of 30 skeletal craniofacial and body traits associated with snail- and scale-eating using lab-reared F2 intercrosses from Crescent Pond and Little Lake. We called variants, estimated linkage maps, and performed QTL analyses independently for each F2 population. We found that only one trait — cranial height—mapped to the same genomic region in both Crescent Pond and Little Lake, but 4 of the 5 remaining significant QTL detected in one lake mapped to the same genomic region as a highly correlated 
130 craniofacial trait in the second lake. Ultimately, we conclude that parallel evolution through

131 reuse of introgressed adaptive alleles is acting to produce similar snail- and scale-eating

132 phenotypes across lake populations on SSI.

\section{Methods}

\section{Genetic cross}

136 Currently, pupfish species can been found in 12 hypersaline lakes across the island: generalist

137 pupfish are found in allopatry in five lakes, the generalist and snail-eating pupfish are found in

138 sympatry without the scale-eater in two lakes, the generalist and scale-eater are found in

139 sympatry in a single lake, and all three species are found in sympatry in four lakes (Martin and

140 Feinstein 2014). We collected wild-caught scale-eating and snail-eating pupfishes from two

141 different sympatric populations (containing all three species) on SSI - Crescent Pond and Little

142 Lake - during the years of 2011 and 2013-2015 using seine nets. We brought individuals back to

143 the University of California, Davis or the University of California, Berkeley and a single wild-

144 caught scale-eating female from each lake was allowed to breed freely with a single wild-caught

145 snail-eating male from the same lake resulting in two separate genetic crosses (one cross from

146 Crescent Pond and one cross from Little Lake). At least four F1 offspring from each hybrid

147 population were crossed to produce F2 intercrosses, resulting in 354 individuals from Crescent

148 Pond and 287 individuals from Little Lake included in this study. All fish were maintained in 40-

149 L tanks at 5-10ppt salinity at the University of California, Davis or the University of California,

150 Berkeley. We fed fry a diet of newly hatched Artemia nauplii for approximately 40 days post

151 fertilization, after which they were switched to the adult diet of frozen and pellet foods. We

152 euthanized fish in an overdose of MS-222 (Finquel, Inc.) according to the approved University of 
153 California, Davis Institutional Animal Care and Use Protocol \#17455 or University of California,

154 Berkeley IACUC Protocol AUP-2015-01-7053, and stored them in 95\% ethanol.

\section{Phenotyping}

\section{Sex and Mate Preferences}

158 For individuals from Crescent Pond, we recorded their sex using their sexually dimorphic body

159 and fin coloration. Male pupfish develop a blue iridescent coloration along their anteriodorsal

160 surface and a black marginal band along their caudal fin (Echelle and Echelle 2020).

161 Once F2 hybrids reached sexual maturity, we performed mating assays using a subset of

162 the hybrid females from Crescent Pond to estimate mate preferences for snail- or scale-eating

163 mates $(\mathrm{N}=74)$. Prior to the mating assays, female fish were isolated for at least twelve hours and

164 conditioned on frozen bloodworms with a 12:12 light:dark cycle. Mating assays occurred in three

$1651.1 \mathrm{~m}$ diameter kiddie pools (5-10ppts salinity). Pools were covered with gravel substrate and

166 divided in half. In each half, we placed three clear plastic 7.5-L Kritter Keepers in a row

167 containing three conspecific males housed individually to avoid aggression. Size-matched scale-

168 eater males were placed on one side of each arena and snail-eating males on the other. Once the

169 males were placed in individually in clear boxes, a female F2 hybrid from Crescent Pond was

170 placed into the center of one of the three kiddie pools, chosen at random. We considered females

171 acclimated to the pool once they had visited both rows of males, after which we started the

172 seven-minute trial period. During each trial we recorded the amount of time a female spent

173 within one body-length of each species. Each female was tested consecutively in all three pools,

174 and we used the mean of her association time (scale-eater association time / total association 
175 time during each 7-minute trial) across all three pools for QTL analysis. Size-matched males

176 were periodically rotated into and among kiddie pools during the 12-month testing period.

\section{Morphological Traits}

179 To measure skeletal phenotypes in our F2 intercrosses, we cleared and double-stained each

180 specimen with alizarin red and alcian blue. Before clearing and staining, each fish was skinned

181 and fixed in $95 \%$ ethanol. We then fixed specimens in $10 \%$ buffered formalin for at least one

182 week and stained batches of individually labeled specimens following Dingerkus and Uhler's

183 (1977) protocol. We suspended cleared and stained specimens in glycerin, and photographed

184 their left lateral side using a Canon EOS 60D digital SLR camera with a $60 \mathrm{~mm}$ macro lens. For

185 each individual, we took two types of photographs: first, we took a whole-body photograph to

186 calculate fin and body measurements and second, a lateral skull image to calculate craniofacial

187 measurements (Figure 1). We used DLTdv8 software (Hedrick 2008) to digitize 11 landmarks

188 on each whole body image and 19 landmarks on each lateral skull image following the

189 morphometric methods described in Martin et al. (2017). For individuals from Crescent Pond, we

190 also weighed the adductor mandibulae muscle mass. Each image included a standardized grid

191 background which we used to calibrate and transform our measurements from pixels into

192 millimeters. In total, we measured 354 individuals from Crescent Pond and 287 individuals from

193 Little Lake. We used R to convert the 30 landmarks into linear distances. To reduce

194 measurement error due to the lateral positioning of the specimens, we took the mean distances

195 from the two clearest skull and whole-body photographs for each individual when possible. If an

196 individual did not have two clear photographs for each orientation, we measured the single

197 clearest photograph. Finally, we size-corrected each trait by using the residuals from a linear 
model including the log-transformed measurement of each trait as the response variable and log-

199 transformed standard length as the predictor variable. We investigated whether size-corrected

200 traits varied between the two populations using a PCA and a MANOVA test, but found no

201 appreciable difference between them (Fig. S1, num df $=28$, approximate $F$-value $=0.34, P=1$ )

\section{Genotyping}

204 We genotyped individuals using three different methods: First, we used whole genome

205 resequencing for the wild-caught F0 parental generation of our Crescent Pond and Little Lake

206 intercrosses. We used DNeasy Blood and Tissue Kits (Qiagen, Inc.) to extract DNA from the

207 muscle tissue of each fish and quantified it on a Qubit 3.0 fluoromether (Thermofisher Scientific,

208 Inc.). Genomic libraries were then prepared at the Vincent J. Coates Genomic Sequencing Center

209 (QB3) using the automated Apollo 324 system (WaterGen Biosystems, Inc.). Samples were

210 fragmented using Covaris sonication and barcoded with Illumina indices. A quality check was

211 also performed on all samples using a Fragment Analyzer (Advanced Analytical Technologies,

212 Inc.). We used 150 paired-end sequencing on an Illumina Hiseq4000 for these four parental

213 samples along with an additional 38 samples that were included in a previous study (Richards

214 and Martin 2017).

215 Second, in addition to the 190 previously sequenced individuals from Crescent Pond used

216 for a QTL mapping study (Martin et al. 2017), we included an additional 164 F2 individuals

217 from Crescent Pond sequenced using double-digest restriction site associated sequencing

218 (ddRADseq) following similar library prep and sequencing methods described in Martin et al.

219 (2015, 2016, 2017). Briefly, we prepared four indexed libraries each containing 96 barcoded 
220 individuals. We sequenced these using 100 single-end high-output mode on two lanes of

221 Illumina Hiseq4000 at the Vincent J. Coates Genomic Sequencing Center (QB3).

222 Finally, we sequenced all F2 individuals from Little Lake and a subset of previously

223 sequenced, but low-coverage Crescent Pond F2's (N=84), using Nextera-tagmented reductively-

224 amplified DNA (NextRad) sequencing (Russello et al. 2015). We followed the above methods

225 for DNA extraction and sent samples to SNPsaurus (SNPsaurus, LLC) for quality checking,

226 NextRad library preparation, and 150 single-end sequencing on two lanes of Illumina Hiseq4000

227 at the University of Oregon sequencing core.

228

229 Calling Variants

230 We used the following methods to call variants separately for: 1$)$ the Crescent Pond intercross (2

231 parents and 354 F2 hybrids), and 2) the Little Lake intercross (2 parents and 285 F2 hybrids):

232 First, we inspected raw read quality using FastQC (Babraham Bioinformatics Institute, v0.11.7)

233 and trimmed reads to their appropriate length (100bp for samples sequenced with ddRAD, and

234 150bp for samples sequenced with NextRAD) using TrimGalore! (v0.6.4). For samples that were

235 sequenced using both ddRAD and NextRad methods, we concatenated trimmed raw reads into a

236 single file. We next used bwa-mem to map reads from all individuals in an intercross, both

237 parents and offspring, to the Cyprinodon brontotheroides reference genome (v 1.0; total

238 sequence length $=1,162,855,435 \mathrm{bp}$; number of scaffolds $=15,698$, scaffold N50 = $32 \mathrm{Mbp}$;

239 (Richards et al. 2021)). We identified duplicate reads using MarkDuplicates and created BAM

240 indices using BuildBamIndex in the Picard package (http://picard.sourceforge.net(v.2.0.1)).

241 Following the best practices guide from the Genome Analysis Toolkit (v 3.5; (Depristo et al.

242 2011)), we called and refined our single nucleotide polymorphism (SNP) variant data set using 
243 the program HaplotypeCaller. Pupfish lack high-quality known alleles because they are a non-

244 model organism; we therefore used the recommended hard filter criteria (QD < 2.0; FS <; 60;

245 MQRankSum <-12.5; ReadPosRankSum <-8; (Depristo et al. 2011; Marsden et al. 2014)) to

246 filter our SNP variant dataset. Ultimately, we detected 13.7 million variants in our Crescent Pond

247 dataset and 14.4 million variants in our Little Lake dataset.

248 We used the program STACKS to further filter our dataset and convert our vcf files into

249 phenotype and genotype comma-separated values files that could be imported into the Rqtl

250 program. Specifically, we used the populations program to filter out variants that were not

251 present in both the parental and F2 populations, and to filter out variants found in $10 \%$ or less of

252 the population. From this filtering step we retained 36,318 variants with 46.5 mean mappable

253 progeny per site in Crescent Pond and 87,579 variants with 85.984 mean mappable progeny per

254 site in Little Lake.

We continued to filter our datasets using the Rqtl (v1.46-2), and ASMap (v1.0-4)

256 packages (Broman et al. 2003; Taylor and Butler 2017). We started filtering by removing

257 individuals that did not contain any filtered variants and any duplicate individuals. This reduced

258 our Crescent Pond data set to 227 individuals, and our Little Lake data set to 281 individuals.

259 Next, we filtered markers that had $>0.98$ or $<0.1$ heterozygosity (Crescent Pond: markers

$260=15,247$, Little Lake: markers=14,661). This step also filtered out 13 individuals from Crescent

261 Pond which only contained markers with $>0.98$ or $<0.1$ heterozygosity. Before constructing our

262 genetic maps, we set aside markers that appeared to suffer from segregation distortion. We used

263 the pullCross() function from the ASmap package to set aside markers in both data sets that were

264 missing in $>75 \%$ of individuals, departed from Mendelian ratios (1:2:1), or any co-located

265 markers for the initial construction of the linkage maps. This filtering retained more than twice 
the number of markers for Crescent Pond than Little Lake. We therefore used a stricter filtering

267 threshold for missing data (i.e., removing markers with $>72 \%$ missing data) for our Crescent

268 Pond dataset to construct linkage maps of comparable sizes for downstream comparative

269 analyses. At the end of this filtering process the Crescent Pond dataset contained 214 individuals

270 and 657 markers and the Little Lake dataset contained 281 individuals with 490 markers.

\section{Linkage Map Construction}

273 We used the mstmap.cross() function to form initial linkage groups and order markers, using the

274 kosambi method for calculating genetic distances and a clustering threshold of $P=1 \times 10^{-14}$ for

275 Little Lake and $P=1 \times 10^{-20}$ for Crescent Pond. After forming these initial linkage groups, we

276 used the pushCross() function from the ASmap package to integrate previously set aside markers

277 back into our map. We pushed markers back based on a segregation ratio of 3:4:3 and we pushed

278 back any markers that had previously been designated as co-located. This increased our map

279 sizes to 817 markers for Crescent Pond and 580 markers for Little Lake. With these additional

280 markers, we re-estimated our linkage map using the est.rf() and formLinkageGroups() functions

281 from the Rqtl package. We used a max recombination fraction of 0.35 and a minimum LOD

282 threshold of 5 to estimate linkage groups for both data sets. We used the

283 droponemarker() command from Rqtl with an error probability of 0.01 to identify and drop

284 problematic markers from the genetic maps, including dropping linkage groups with 3 or fewer

285 markers. Finally, we visually inspected our linkage groups using plotRF() from the Rqtl package,

286 and merged linkage groups which had been incorrectly split up using the mergeCross() function

287 from the ASmap package. Ultimately our final genetic maps included: 1) Crescent Pond: 214 
individuals, 743 markers, 24 linkage groups and 2) Little Lake: 281 individuals, 540 markers,

289 and 24 linkage groups (Figure 2).

\section{QTL Analyses}

292 We mapped QTL for 29 skeletal traits for both populations, and additional morphological

293 (adductor mandibulae muscle mass) and behavioral traits (mate preference) for Crescent Pond.

294 We used the Rqt12 package (v0.22-11) to calculate genotype probabilities with a multipoint

295 hidden Markov model using an error probability of 0.0001 and a Kosambi map function. We

296 calculated kinship matrices to account for the relationship among individuals in two ways: 1)

297 overall kinship, which represents the proportion of shared alleles between individuals, and 2)

298 kinship calculated using the leave-one-chromosome-out method (LOCO). We used the scan1()

299 function to perform three separate genome scans using a single-qtl model by: 1) Haley-Knott

300 regression, 2) a linear mixed model using the overall kinship matrix, and 3) a linear mixed model

301 using the LOCO kinship matrix. For our Crescent Pond data set we also included sex as an

302 additive covariate. We assessed the significance of all three models using two significance

303 thresholds $P<0.1$ and 0.05 based on 1000 permutations each, using the scan1perm() function.

304 As noted above the scan1() function can use several different methods to determine if a region is

305 significantly associated with a given phenotype (Broman et al., 2019; Haley \& Knott, 1992;

306 Yang, Zaitlen, Goddard, Visscher, \& Price, 2014; Yu et al., 2006), however, it is clear from

307 previous theoretical work that many of these methods may suffer from type II error depending on

308 the size of an organism's genome, the density of markers in a linkage map, or the complexity of

309 the phenotypic traits being measured (Lander and Botstein 1989; Risch 1990). We therefore

310 relaxed the $P$-value cut off from 0.05 to 0.1 to capture potentially important genomic regions. 
311 This relaxation is further supported by the LOD scores associated with regions significant at the

$312 P<0.1$ level because they all exceed the traditional threshold of 3 (Nyholt 2000), the more

313 conservative threshold of $~ 3.3$ (Lander and Kruglyak 1995; Nyholt 2000), the suggestive

314 threshold of 1.86 (Lander and Kruglyak 1995), and are in line with estimates of significant LOD

315 thresholds in previous studies (Erickson et al. 2016). All three of these methods detected similar

316 QTLs and moving forward we only used the Haley-Knott regression method.

317 For each trait, we calculated the location of the maximum LOD score, and used the fit1()

318 function to re-fit a single-QTL model at this location. We used the newly calculated LOD score

319 to estimate the proportion of variance explained by the QTL and to calculate a $P$-value

320 associated with each significant QTL ( $x^{2}$-test). We also used the location of the maximum LOD

321 score to calculate $95 \%$ Bayes credible intervals using the bayes_int() function from the Rqt12

322 package. We note that the maximum LOD score associated with every trait across both ponds

323 exceeded the suggestive threshold of 1.86 (Lander and Kruglyak 1995). We used the

324 find.markerpos() function from Rqtl to determine where markers in each linkage map fell within

325 the reference genome. With this information we were able to determine the scaffolds/positions

326 from the reference genome that fell within the 95\% credible intervals for each putative QTL.

327 Finally, we used the maxmarg() function from the Rqt12 package to find the genotype with the

328 maximum marginal probability at the location of the maximum LOD. We used these genotypes

329 to visualize the relationship between genotype and phenotypes.

\section{Identifying adaptive alleles within QTL regions}

332 For each scaffold that fell within a QTL's credible interval we calculated the minimum and

333 maximum position for that scaffold (that was identified in the putative QTL region) and searched 
334 the C. brontotheroides reference genome for annotated genes within the region. We then

335 compared this list to a previously published list of genes that 1) contained or were adjacent to

336 (within $20 \mathrm{kbp}$ ) fixed or nearly fixed (Fst > 0.95) SNPs between specialist species on SSI, and 2)

337 showed significant evidence of a hard selective sweep in both the site frequency spectrum-based

338 method SweeD (Pavlidis et al. 2013) and the linkage-disequilibrium-based method OmegaPlus

339 (Alachiotis et al. 2012). We hereafter refer to these loci as adaptive alleles. We also noted

340 whether adaptive alleles within QTL regions were classified as de novo, introgressed, or as

341 standing genetic variation on SSI (Richards et al. 2021). We used a bootstrap resampling method

342 to determine whether the observed proportions of adaptive alleles originating from de novo,

343 introgression, or standing genetic variation found within QTL 95\% credible intervals were

344 different than the proportions expected when drawn from the genome at random. We used the

345 boot (v. 1.3-25) package (Buckland et al. 1998; Canty and Ripley 2021) to resample our entire

346 adaptive allele dataset (with replacement) 10,000 times. We then used the boot.ci() command

347 from the boot package to calculated the $95 \%$ credible intervals around expected proportions of de

348 novo, introgressed, and standing adaptive alleles. We performed these calculations separately for

349 scale-eater and snail-eater adaptive alleles.

$351 \quad$ Results

352 Linkage Map Construction

353 We identified 24 linkage groups from 743 markers for Crescent Pond, and 24 linkage groups

354 from 540 markers for Little Lake (Figure 2). Previous karyotypes of Cyprinodon species

355 estimated 24 diploid chromosomes, matching the linkage groups in this study (Liu \& Echelle,

356 2013; Stevenson, 1981). The total map length for Crescent Pond was $7335 \mathrm{cM}$ and the total map 
357 length for Little Lake was 5330; the largest linkage groups for each map were $740 \mathrm{cM}$ and 380

$358 \mathrm{cM}$, respectively, and inter-marker map distance did not exceed 20cM in either map. To compare

359 our maps and to determine if the same genomic regions were being reused across lakes, we

360 identified where each marker was located in our reference genome. Overall, we found 324

361 markers in both maps that were within $10 \mathrm{Kbp}$ of one another, indicating that $60 \%$ of the Little

362 Lake map was also present in the Crescent Pond map and 44\% of the Crescent Pond map was

363 present in the Little Lake map (Figure 3).

\section{Craniofacial QTL}

366 We detected three significant QTL in Crescent Pond and five QTL in Little Lake (Table 1,

367 Table 2). In Crescent Pond, we identified QTL associated with the depth of the dentigerous arm

368 of the premaxilla, cranial height, and adductor mandibulae muscle mass. Cranial height in

369 Crescent Pond mapped to linkage group (LG) 10. Dentigerous arm depth and adductor

370 mandibulae muscle mass both mapped to LG 13, which also contained the max LOD scores for

371 two additional jaw traits (jaw opening in-lever and maxillary head height; Table 2). The $95 \%$

372 credible intervals for all these traits overlapped, suggesting that LG 13 may contain a single

373 pleiotropic locus or many loci that affect all four traits.

374 In Little Lake, we detected significant QTL associated with jaw closing in-lever (i.e.

375 height of the coronoid process on the articular: LG9), width and depth of the dentigerous arm of

376 the premaxilla (LG3 and LG6), maxillary head protrusion (LG10), and cranial height (LG1;

377 Table 1,Table 2). The 95\% credible interval for dentigerous arm width on LG3 also contained

378 the max LOD score for lower jaw length, suggesting that either a single pleiotropic locus or a

379 cluster of loci in this region may be controlling both traits. 


\section{Candidate genes and adaptive alleles within QTL regions}

\section{Cranial height}

383 Cranial height was the only trait with statistically significant or marginally significant QTL in

384 both lakes (Figure 4, $P<0.1$ ). While the QTL occurred on different linkage groups between

385 maps, we found a high degree of synteny between these linkage groups indicating that the QTL

386 is located in the same genomic region in both lakes (Table 2, Figure 3). We also found the

387 same overdominant genetic pattern in both lake crosses: heterozygotes showed increased cranial

388 height relative to homozygous individuals (Figure 5).

We found 44 genes within scaffold 33 that fell partially or fully within the $95 \%$ credible

390 intervals of the QTL in both lakes (Table 1, Table S1). Only three of these genes contained

391 adaptive alleles within $20 \mathrm{~kb}$ : wdr31, bri3bp, and gnaq (Table 3). Interestingly, gnaq is well

392 known to be associated with craniofacial development (Hall et al. 2007; Shirley et al. 2013) and

393 is differentially expressed between our specialist species in developing larvae (McGirr and

394 Martin 2020).

\section{Dentigerous Arm Width}

397 We found that regions on scaffolds 58 and 24 were associated with a significant QTL for

398 dentigerous arm width in Little Lake and contained the max LOD scores for maxillary head

399 protrusion and female mate preference in Crescent Pond (Table 1, Table 2). We found 161

400 genes which fell partially or completely within these shared regions, but only 2 genes, $d y s f$ and

401 cyp26b1, which contained adaptive alleles within $20 \mathrm{kbp}$ (Table 3). The $d y s f$ gene provides

402 instructions for making a protein called dysferlin, which is found in the sarcolemma membrane 
that surrounds muscle fibers (Liu et al. 1998). This could indicate a role for muscle development

404 in affecting skeletal development of the maxilla and premaxilla.

405

406 Dentigerous Arm Depth

407 The QTL for dentigerous arm depth in Little Lake was associated with LG 6, which corresponds

408 to LG 7 in Crescent Pond, however, no traits from Crescent Pond mapped to this linkage group

409 (Table 2, Figure 3). Instead, dentigerous arm depth in Crescent Pond was associated with LG 13

410 and did not share any similar genomic regions with those associated with dentigerous arm depth

411 in Little Lake. We found 80 genes completely or partially within the $95 \%$ credible region for this

412 QTL in Little Lak, but none contained adaptive alleles based on our criteria (Figure S1). In fact,

413 only a single adaptive allele was found in this QTL region, but it was in an unannotated region of

414 the genome (Table 3).

Maxillary Head Protrusion

417 Maxillary head protrusion in Little Lake mapped to a QTL region on LG10 which corresponds to

418 the max LOD scores for both lower jaw length and caudal peduncle height in Crescent Pond

419 (Table 2, Figure 3). Across lakes, all three traits were associated with scaffolds 53, 2336, and

420 6275. We found 528 genes partially or fully within these shared regions, but only 21 of these

421 genes contained adaptive alleles within $20 \mathrm{kbp}$ (Table 3). One of these genes, twist 1 , contains a

422 non-synonymous substitution fixed in scale-eating pupfish on San Salvador Island, Bahamas

423 (Richards et al. 2021). Twistl is a transcription factor and oncogene associated with palate

424 development and oral jaw size in model organisms (Parsons et al. 2014; Teng et al. 2018). 
427 The QTL for jaw closing in-lever was associated with LG 9 in Little Lake, which corresponds to

428 the max LOD scores for orbit diameter and anterior body depth in Crescent Pond (Table 2,

429 Figure 3). Scaffolds 8 and 8020 were associated with all three of these traits. We found 13 genes

430 which partially or completely fell within these shared regions, and only two genes, map $2 k 6$ and

431 galr2, which contained adaptive alleles within $20 \mathrm{kbp}$ (Table 3). Galr2 was also previously

432 detected within a significant QTL for lower jaw length in pupfish (Martin et al. 2017).

434 Dentigerous Arm Depth and Adductor Mandibulae Muscle Mass

435 Finally, in Crescent Pond the QTL for dentigerous arm depth and adductor mandibulae muscle

436 mass mapped to the exact same location on LG 13 (95\% CI dentigerous arm depth (0, 250),

437 adductor mandibulae muscle mass $(0,70)$. This linkage group corresponds to LG14 in Little

438 Lake, which contains the max LOD scores for both palatine height and suspensorium length

439 (Table 3). We found 52 genes that overlapped between these regions, 18 of which contained

440 adaptive alleles. Furthermore, three of the genes- ube $2 w$, ncoa2, and prlh — contained adaptive

441 alleles that introgressed from Laguna Bavaro in the Dominican Republic to snail-eating pupfish

442 (ube2w), from Lake Cunningham, New Providence Island to scale-eating pupfish (ncoa2), or

443 from North Carolina, USA to scale-eating pupfish ( $p r l h)$. We also found four genes that

444 contained adaptive alleles within $20 \mathrm{kbp}$ that arose from de novo mutations: $c d 226, \mathrm{cmbl}$, slc51a,

445 and $z$ fhx; however, only one adaptive allele in slc5la is found within a coding region. 
Adaptive alleles originating from standing genetic variation across the Caribbean were most

449 common within shared QTL regions between lakes (86.03\% within scale-eater populations, and

$450 \quad 53.32 \%$ within snail-eating populations; Table 3). However, observed proportions within shared

451 QTL were significantly less than expected by chance (scale-eater expected 95\% CI: (88.33\%-

$45290.37 \%)$, snail-eater expected 95\% CI: (62\%-67\%;10,000 bootstrapped iterations). Instead, we

453 found more introgressed scale-eater and snail-eater adaptive variants in shared QTL regions than

454 expected by chance (Scale-eater observed: 12.13\% introgressed, scale-eater expected 95\% CI:

455 (7.96\%-9.88\%); Snail-eater observed: $46.67 \%$ introgressed, snail-eater expected 95\% CI:

$456(32.22 \%-37.06 \%))$. Finally, we found that about $1.83 \%$ of adaptive alleles within overlapping

457 regions between lakes originated from de novo mutations in scale-eaters, however, this fell

458 within the predicted null range (95\% CI: (1.29\%-2.17\%)).

460 Discussion

\section{Parallel genetic changes underlie 5 out of 6 of craniofacial QTL}

462 We found evidence supporting both parallel and non-parallel genetic changes in an adaptive

463 radiation of trophic specialist pupfishes. A single significant QTL was associated with cranial

464 height in both lakes and mapped to the same genomic region, suggesting that parallel genetic

465 changes are responsible for variation in this trait in both lakes. On the other hand, significant

466 QTLs were identified for premaxilla dentigerous arm depth in each lake, but they mapped to

467 different locations, indicating that this trait is associated with non-parallel genetic changes. We

468 found an additional three traits with significant QTLs (dentigerous arm width, jaw closing in-

469 lever, maxillary head protrusion) in the Little Lake population that were not detected in Crescent

470 Pond. However, all genomic regions associated with these traits in Little Lake also mapped to 
471 the max LOD score for this same integrated suite of craniofacial traits in Crescent Pond.

472 Therefore, rather than assume independent QTL for each trait, we conservatively conclude that

473 the same genomic regions are being reused in each lake and affect a highly integrated suite of

474 craniofacial traits. Overall, we found that 5 out of the 6 significant QTLs were reused in some

475 way across lakes suggesting that parallel genetic changes underly adaptive phenotypes in the San

476 Salvador Island pupfish radiation.

\section{High level of QTL reuse across ponds}

479 Overall, we found that about 16\% (1 out of 6) of the identified QTL regions corresponded to 480 non-parallel changes and 84\% (5 out of 6) corresponded to parallel genetic changes-either

481 affecting the same phenotypic trait or a tightly correlated craniofacial trait- across populations.

482 The presence of both non-parallel and parallel genetic changes leading to convergent phenotypes

483 across lakes has been documented previously. For example, Colosimo et al. (2004) investigated

484 the genetic basis of armor plate morphology in two independent threespine stickleback

485 populations and found a single large effect locus on LG 4 in the two populations. However, they

486 also noted a potential difference in the dominance relationships of alleles across ponds at this

487 location, and found additional differences in modifier QTLs between populations, suggesting

488 that both parallel and non-parallel genetic changes could lead to the loss of armor plating.

489 Similarly, Erickson et al. (2016) found evidence for both parallel (43\% of QTL regions

490 overlapped between at least two populations) and non-parallel (57\% of QTL regions were found

491 in only a single population) evolution in a QTL study investigating the genetic basis of 36

492 skeletal phenotypes in three independent threespine stickleback populations. However, our

493 findings suggest that pupfish exhibit a much higher proportion of parallel evolution than 
494 previously documented in stickleback. In fact, Conte et al. (2012) estimated that the probability

495 of convergence via gene reuse is only $32-55 \%$ — which is 1.5 to 2.5 times lower than our current

496 finding — although this may be underestimated (Stern 2013).

497 Pupfish may have a higher rate of parallel evolution than other model fish speciation

498 systems for a few reasons. First, the pupfish radiation is recent, although comparable in age to

499 glacial stickleback populations, with specialist species diverging less than 10kya (Hagey and

500 Mylroie 1995; Martin and Wainwright 2013), and parallel evolution is predicted to be more

501 likely when populations or species have recently diverged (Rosenblum et al. 2014). This may be

502 because recently diverged species are more likely to experience similar environments, have

503 access to similar pools of genetic variation (either due to standing genetic variation or

504 introgression), or similar genetic constraints. Second, the genomic basis of pupfish skeletal traits

505 may be primarily controlled by cis-regulatory elements, which evolve more quickly and have

506 less negative pleiotropy which may make them more likely to undergo parallel evolution (Stern

507 and Orgogozo 2008). However, a previous study of allele-specific expression in the pupfish

508 system found strong evidence that two cis-regulatory alleles were associated with skeletal

509 development, but trans-acting elements predominated overall (McGirr and Martin 2021).

510 In part, the increased proportion of parallel evolution estimated in this study results from

511 our relaxed thresholds for detecting and categorizing shared QTL regions. Previous QTL studies

512 have typically searched for evidence of parallel evolution by only looking for one-to-one

513 mapping in which the same genomic regions are associated with the same trait across

514 populations at a genome-wide level of significance in each (Colosimo et al. 2004; Conte et al.

515 2012). While this method provides the most clear-cut examples of parallel evolution, we argue

516 that it vastly underestimates its frequency in nature. For example, this method would not 
517 consider reuse of the same genomic regions for integrated morphological traits as parallel

518 evolution, a pattern seen in this study and in Erickson et al. (2016). Furthermore, the strict one-

519 to-one significance method for detecting parallel evolution does not include consideration of the

520 hierarchy and diversity of convergence and parallel evolution, which can span morphological

521 traits, ecotypes, performance, or even fitness (James et al. 2020; Rosenblum et al., 2014; Stern,

522 2013; Martin and Wainwright 2013). Ultimately, we argue that our method of quantifying

523 parallel evolution provides a more wholistic view of the process and better captures the

524 frequency of reuse of adaptive genetic variation in nature.

525

526 Few QTL may affect many highly integrated craniofacial traits

527 There are several processes that may cause the same genomic regions to be associated with

528 different traits between lakes. First, these genomic regions may be highly pleiotropic and affect

529 several traits simultaneously. For example, Albert et al. (2007) found that that on average a

530 single QTL affected 3.5 phenotypic traits in an analysis of 54 body traits in three-spine

531 stickleback. Wagner et al. (2008) found a similar pattern in QTL analyses of 70 skeletal traits in

532 mice, where a single QTL affected on average 7.8 phenotypic traits (the maximum being 30 ).

533 A second possibility is that a single QTL region may contain several tightly linked

534 causative variants that are responsible for variation in many traits. Correlated phenotypic traits

535 are generally assumed to have a shared genetic basis, but it is extremely difficult to determine if

536 this is due to pleiotropy or tight linkage between genomic regions (Lynch and Walsh 1998;

537 Gardner and Latta 2007; Paaby and Rockman 2013; Wright et al. 2010).

538 Finally, it may be that differences in methodology or sample sizes between lakes enable

539 us to detect significant QTL for some traits in one lake and not the other. For example, our 
540 analyses of Little Lake allowed us to detect significant QTL for effect sizes greater than 6.54

541 PVE at $80 \%$ power, but we could only detect significant QTL for effect sizes greater than 8.41

542 PVE at $80 \%$ power in Crescent Pond due to our lower sample size for this cross (Sen et al.

543 2007). However, this level of power is typical in many non-model QTL studies (Ashton et al.

544 2017). The ability to detect a significant QTL in one lake but not the other may be further

545 explained by our use of different sequencing methods between populations. However, a critical

546 component of our analyses involved searching for regions within $10 \mathrm{kbp}$ of one another across

547 maps to provide confidence that if we detected a significant QTL in one lake and not the other

548 that it was not simply because that genomic region was not captured by the sequencing. For

549 example, in Little Lake we detected a significant QTL associated with dentigerous arm depth on

550 LG 6 but did not find any traits associated with this region of the genome in Crescent Pond.

552 QTL are associated with different craniofacial traits across different lakes

553 In this study we found an intriguing pattern of different traits mapping to the same region of the

554 genome across lake populations. One potential explanation for this is that there are different

555 relationships between traits in each lake, and we find some evidence of this in our phenotypic

556 data. Figure S2 depicts correlation matrices between traits in 1) Little Lake and 2) Crescent

557 Pond, and $X^{2}$ comparisons of these two matrices reveals that the relationship between traits

558 varies significantly between lakes $\left(X^{2}=3135.99, \mathrm{df}=756, P<3.6 \mathrm{e}-29\right)$. For example, the

559 relationship between maxillary head protrusion and lower jaw length is more than two times

560 stronger in Little Lake compared to Crescent Pond (Pearson's $r_{L L}=0.27$, Pearson's $r_{C P}=0.12$ ),

561 the relationship between dentigerous arm depth and suspensorium length is 1.8 times stronger in

562 Little Lake than in Crescent Pond (Pearson's $r_{L L}=0.45$, Pearson's $r_{C P}=0.24$ ), and the relationship 
563 between jaw closing in-lever and anterior body depth is more than two times stronger in Crescent

564 Pond than in Little Lake (Pearson's $\mathrm{r}_{\mathrm{CP}}=0.23$, Pearson's $\mathrm{r}_{\mathrm{LL}}=0.11$ ).

565 This pattern may be explained by different epistatic interactions in each lake. For

566 example, Juenger et al. (2005) detected significant QTL-QTL interactions in one mapping

567 population of Arabidopsis but found no evidence of the same interactions in the other population.

568 When we investigated the relationship between phenotype and genotype for cranial height, we

569 found the same overdominance pattern in both lakes (Figure 5). However, the presence of

570 epistatic interactions may also be an obstacle for QTL detection. In a mapping study of body

571 weight in chicken, Carlborg et al. (2006) were only able to detect a single weak QTL despite the

572 extremely divergent phenotypes between parental lines. However, when accounting for epistatic

573 interactions, Carlborg et al. identified several additional significant QTL regions that explained a

574 large amount of variation in body weights.

575 Finally, our method for searching for putative QTL regions may have led to this pattern.

576 Similar studies have searched for influential genomic regions by first identifying a putative QTL

577 in a single population, and then searching the already identified linkage group in the second

578 population for any signal of a QTL associated with the same phenotype, often using relaxed

579 LOD thresholds closer to the suggestive cut-off (LOD> 1.8, e.g., Erickson et al. 2016). Our

580 approach, however, independently identified the positions of maximum LOD for all traits across

581 the entire linkage map before searching for similar implicated regions between populations. We

582 argue that our approach minimizes bias, because there are no prior expectations about which

583 traits should be associated with a given genomic region within a suite of integrated traits, and

584 reduces false positives because we only examine the maximum LOD position for each trait. 


\section{Identifying causative regions within QTL}

587 Multiple mapping populations across lakes may also be particularly useful for identifying

588 candidate causal alleles. We found that one out of our six unique QTL regions mapped to the

589 same genomic location across lakes and was associated with the same phenotypic trait—cranial

590 height (Figure 4). In Crescent Pond, we found that a region of $110 \mathrm{cM}$ was associated with this

591 trait (LG10, position: 204, 95\% CI (130,340)), which contained 426 genes. However, when we

592 compared this region to the region independently identified in our Little Lake analysis, we found

593 that the overlapping region was reduced to 20cM (LG1, position: $259,95 \% \mathrm{CI}(250-270)$ ) and

594 contained only 44 genes - a reduction of more than $80 \%$. We found a similar pattern in the

595 additional four QTL regions that mapped to the same genomic location across maps but were

596 associated with different phenotypic traits and observed an average $56 \%$ reduction in region size.

597 As noted above, Erickson et al. (2016) used a similar method of identifying candidate QTL

598 regions across three hybrid populations of stickleback, and found that $43 \%$ of identified QTL

599 regions were shared across two or more populations; however, they did not investigate whether

600 these QTL regions completely or partially overlapped.

$601 \quad$ We also searched for adaptive alleles within QTL region that were identified in a

602 previous study as 1) nearly fixed between species $\left(\mathrm{F}_{\mathrm{st}}>0.95\right)$ and 2$)$ showed significant evidence

603 of a hard selective sweep (Richards et al. 2021). Overall, we found 789 shared genes within

604 shared QTL regions across lakes, and that 45 of those genes contained adaptive variants (5.7\%).

605 This is a six-fold increase from the genome-wide expectation of $0.91 \%$ (176 genes associated

606 with at least one adaptive variant / 19304 annotated genic regions), suggesting that these specific

607 regions are important for adaptation to scale- and snail-feeding in wild pupfish. For example, a

608 variant in twist 1 was found within the region associated with maxillary head protrusion in Little 
609 Lake (which also overlapped with lower jaw length and caudal peduncle height in Crescent

610 Pond). In model organisms, twist1 is associated with palate and jaw development (Parsons et al.

611 2014; Teng et al. 2018), and previous genome-wide association scans in pupfish showed that a

612 region containing twist 1 was significantly associated with oral jaw size in the system (Richards

613 et al. 2021). Similarly, we found that variants associated with galr2 fell within the QTL region

614 associated with jaw closing in-lever in Little Lake (which also overlapped with regions

615 associated with orbit diameter and anterior body depth in Crescent Pond; scaffolds 8 and 8020),

616 and previous QTL mapping studies, gene expression studies, and genome-wide association

617 analyses have all implicated regions containing galr2 with oral jaw development in pupfish

618 (McGirr and Martin 2016; Martin et al. 2017; Richards et al. 2021).

Increased use of introgressed adaptive variants in QTL regions

621 We found that most genetic variation within shared QTL regions was also segregating across

622 outgroup Caribbean generalist populations characterized by Richards et al. $(2021 ; 86.04 \%$ within

623 scale-eater populations, and $53.32 \%$ within snail-eating populations). Furthermore, we found

624 more introgressed adaptive alleles from both scale-eater (observed: $12.13 \%$ introgressed,

625 expected 95\% CI: (7.96\%-9.88\%)) and snail-eater populations in shared QTL regions than

626 expected by chance (observed: 46.67\%, expected 95\% CI: (32.22\%-37.06\%)). This supports the

627 prediction that standing genetic variation and introgressed variation should underlie parallel

628 genetic changes (Stern 2013; Thompson et al. 2019). Finally, we found that only $1.83 \%$ of

629 adaptive alleles within shared QTL regions across both lakes originated from de novo mutations

630 on San Salvador Island. While this percentage did not differ significantly from the expected 
631 estimates (expected 95\% CI: $1.3 \%-2.17 \%$ ) it does not eliminate the possibility that de novo

632 mutations play an important adaptive role in pupfish evolution.

\section{Conclusion}

635 In conclusion, we found that a single QTL region was responsible for variation in cranial height

636 in both populations, and an additional four QTL regions were responsible for variation in

637 different craniofacial traits across lakes, suggesting that parallel genetic changes underlie

638 integrated suites of adaptive craniofacial phenotypes on San Salvador Island. Adaptive alleles

639 were more commonly found within these detected QTL regions, and more of these adaptive

640 alleles arrived on SSI via introgression than expected by chance. Finally, we argue that

641 investigating QTL regions across populations in concert with estimation of hard selective sweeps

642 in wild populations is a powerful tool for identifying potential causative regions of the genome

643 affecting adaptive divergence.

\section{Acknowledgements}

646 We thank the University of California, Davis, the University of California, Berkeley, the

647 University of North Carolina at Chapel Hill, NSF CAREER 1749764, NIH 5R01DE027052-02,

648 and BSF 2016136 for funding to CHM. The Bahamas Environmental Science and Technology

649 Commission and the Ministry of Agriculture provided permission to export fish and conduct this

650 research. Rochelle Hanna, Velda Knowles, Troy Day, and the Gerace Research Centre provided

651 logistical assistance in the field. All animal care protocols were approved by the University of

652 California, Davis and the University of California, Berkeley Animal Care 


\section{Author Contributions}

655 MESJ and CHM designed research; MESJ, CHM, JCD, and SR performed data collection; MESJ

656 and EJR performed data analysis; MESJ and CHM wrote the paper. CHM provided funding.

Data Accessibility

659 Data will be deposited to Dryad and NCBI. Genomes are archived at the National Center for

660 Biotechnology Information BioProject Database (Accessions:

661 PRJNA690558; PRJNA394148, PRJNA391309; and PRJNA305422).

\section{References}

Alachiotis, N., A. Stamatakis, and P. Pavlidis. 2012. OmegaPlus: A scalable tool for rapid detection of selective sweeps in whole-genome datasets. Bioinformatics 28:2274-2275. Oxford Academic.

Albert, A. Y. K., S. Sawaya, T. H. Vines, A. K. Knecht, C. T. Miller, B. R. Summers, S. Balabhadra, D. M. Kingsley, and D. Schluter. 2007. The genetics of adaptive shape shift in stickleback: pleiotropy and effect size. Evolution (N. Y). 0:071115145922005-??? John Wiley \& Sons, Ltd.

Ashton, D. T., P. A. Ritchie, and M. Wellenreuther. 2017. Fifteen years of quantitative trait loci studies in fish: challenges and future directions. Mol. Ecol. 26:1465-1476.

Beavis, W. D. 1998. QTL analyses: power, precision, and accuracy. Pp. 145-162 in Molecular dissection of complex traits.

Besnard, G., A. M. Muasya, F. Russier, E. H. Roalson, N. Salamin, and P. A. Christin. 2009. Phylogenomics of C4 photosynthesis in sedges (Cyperaceae): Multiple appearances and genetic convergence. Mol. Biol. Evol. 26:1909-1919. Oxford Academic.

Bolnick, D. I., R. D. H. Barrett, K. B. Oke, D. J. Rennison, and Y. E. Stuart. 2018. (Non)Parallel evolution. Annual Reviews Inc.

Bricelj, V. M., L. Connell, K. Konoki, S. P. MacQuarrie, T. Scheuer, W. A. Catterall, and V. L. Trainer. 2005. Sodium channel mutation leading to saxitoxin resistance in clams increases risk of PSP. Nature 434:763-767. Nature Publishing Group.

Broman, K. W., D. M. Gatti, P. Simecek, N. A. Furlotte, P. Prins, Ś. Sen, B. S. Yandell, and G. A. Churchill. 2019. R/qt12: Software for mapping quantitative trait loci with highdimensional data and multiparent populations. Genetics 211:495-502. Genetics Society of America. 
Broman, K. W., H. Wu, S. Sen, and G. A. Churchill. 2003. R/qtl: QTL mapping in experimental crosses. Bioinformatics 19:889-890. Oxford Academic.

Buckland, S. T., A. C. Davison, and D. V. Hinkley. 1998. Bootstrap Methods and Their Application. Biometrics 54:795.

Canty, A., and B. D. Ripley. 2021. boot: Bootstrap R (S-Plus) Functions. R Packag. version 1.328.

Carlborg, Ö., L. Jacobsson, P. Åhgren, P. Siegel, and L. Andersson. 2006. Epistasis and the release of genetic variation during long-term selection. Nat. Genet. 38:418-420. Nature Publishing Group.

Chan, Y. F., M. E. Marks, F. C. Jones, G. Villarreal, M. D. Shapiro, S. D. Brady, A. M. Southwick, D. M. Absher, J. Grimwood, J. Schmutz, R. M. Myers, D. Petrov, B. Jónsson, D. Schluter, M. A. Bell, and D. M. Kingsley. 2010. Adaptive evolution of pelvic reduction in sticklebacks by recurrent deletion of a pitxl enhancer. Science (80-. ). 327:302-305. American Association for the Advancement of Science.

Chen, L., A. L. Devries, and C. H. C. Cheng. 1997. Convergent evolution of antifreeze glycoproteins in Antarctic notothenioid fish and Arctic cod. Proc. Natl. Acad. Sci. U. S. A. 94:3817-3822.

Christin, P. A., N. Salamin, V. Savolainen, M. R. Duvall, and G. Besnard. 2007. C4 Photosynthesis Evolved in Grasses via Parallel Adaptive Genetic Changes. Curr. Biol. 17:1241-1247. Cell Press.

Colosimo, P. F., C. L. Peichel, K. Nereng, B. K. Blackman, M. D. Shapiro, D. Schluter, and D. M. Kingsley. 2004. The genetic architecture of parallel armor plate reduction in threespine sticklebacks. PLoS Biol. 2:e109. Public Library of Science.

Conte, G. L., M. E. Arnegard, C. L. Peichel, and D. Schluter. 2012. The probability of genetic parallelism and convergence in natural populations. Proc. R. Soc. B Biol. Sci. 279:50395047. Royal Society.

Cresko, W. A., A. Amores, C. Wilson, J. Murphy, M. Currey, P. Phillips, M. A. Bell, C. B. Kimmel, and J. H. Postlethwait. 2004. Parallel genetic basis for repeated evolution of armor loss in Alaskan threespine stickleback populations. Proc. Natl. Acad. Sci. U. S. A. 101:6050-6055.

Depristo, M. A., E. Banks, R. Poplin, K. V Garimella, J. R. Maguire, C. Hartl, A. A. Philippakis, G. Del Angel, M. A. Rivas, M. Hanna, A. McKenna, T. J. Fennell, A. M. Kernytsky, A. Y. Sivachenko, K. Cibulskis, S. B. Gabriel, D. Altshuler, and M. J. Daly. 2011. A framework for variation discovery and genotyping using next-generation DNA sequencing data. Nat. Genet. 43:491-501.

Dingerkus, G., and L. D. Uhler. 1977. Stain Technology Enzyme Clearing of Alcian Blue Stained Whole Small Vertebrates for Demonstration of Cartilage. , doi: 10.3109/10520297709116780.

Donley, J. M., C. A. Sepulveda, P. Konstantinidis, S. Gemballa, and R. E. Shadwick. 2004. Convergent evolution in mechanical design of lamnid sharks and tunas. Nature 429:61-65.

Echelle, A. A., and A. E. Echelle. 2020. Cyprinodontidae $\square$ : Pupfishes. Pp. 609-673 in Freshwater Fishes of North America. The Johns Hopkins University Press.

Erickson, P. A., A. M. Glazer, E. E. Killingbeck, R. M. Agoglia, J. Baek, S. M. Carsanaro, A. M. Lee, P. A. Cleves, D. Schluter, and C. T. Miller. 2016. Partially repeatable genetic basis of benthic adaptation in threespine sticklebacks. Evolution (N. Y). 70:887-902.

Feldman, C. R., E. D. Brodie, E. D. Brodie, and M. E. Pfrender. 2012. Constraint shapes 
convergence in tetrodotoxinresistant sodium channels of snakes. Proc. Natl. Acad. Sci. U. S. A. 109:4556-4561.

Feldman, C. R., E. D. Brodie, E. D. Brodie, and M. E. Pfrender. 2009. The evolutionary origins of beneficial alleles during the repeated adaptation of garter snakes to deadly prey. Proc. Natl. Acad. Sci. U. S. A. 106:13415-13420.

Feulner, P. G. D., F. J. J. Chain, M. Panchal, C. Eizaguirre, M. Kalbe, T. L. Lenz, M. Mundry, I. E. Samonte, M. Stoll, M. Milinski, T. B. H. Reusch, and E. Bornberg-Bauer. 2013. Genome-wide patterns of standing genetic variation in a marine population of three-spined sticklebacks. Mol. Ecol. 22:635-649.

Futuyman, D. J. 1986. Evolutionary Biology. 2d ed. Sinauer, Sunderland, Mass.

Gardner, K. M., and R. G. Latta. 2007. Shared quantitative trait loci underlying the genetic correlation between continuous traits. Mol. Ecol. 16:4195-4209. John Wiley \& Sons, Ltd.

Grant, P. R., B. R. Grant, J. A. Markert, L. F. Keller, and K. Petren. 2004. Convergent evolution of Darwin's finches caused by introgressive hybridization and selection. Evolution (N. Y). 58:1588-1599. Society for the Study of Evolution.

Haenel, Q., M. Roesti, D. Moser, A. D. C. MacColl, and D. Berner. 2019. Predictable genomewide sorting of standing genetic variation during parallel adaptation to basic versus acidic environments in stickleback fish. Evol. Lett. 3:28-42. Wiley.

Hagey, F. M., and J. E. Mylroie. 1995. Pleistocene lake and lagoon deposits, San Salvador island, Bahamas. Spec. Pap. Soc. Am. 77-90.

Haley, C. S., and S. A. Knott. 1992. A simple regression method for mapping quantitative trait loci in line crosses using flanking markers. Heredity (Edinb). 69:315-324.

Hall, B. D., R. G. Cadle, S. M. Morrill-Cornelius, and C. A. Bay. 2007. Phakomatosis pigmentovascularis: Implications for severity with special reference to Mongolian spots associated with Sturge-Weber and Klippel-Trenaunay syndromes. Pp. 3047-3053 in American Journal of Medical Genetics, Part A. John Wiley \& Sons, Ltd.

Hedrick, P. W. 2013. Adaptive introgression in animals: Examples and comparison to new mutation and standing variation as sources of adaptive variation. John Wiley \& Sons, Ltd.

Hedrick, T. L. 2008. Software techniques for two- and three-dimensional kinematic measurements of biological and biomimetic systems. Bioinspiration and Biomimetics 3.

Hernandez, L. P., D. Adriaens, C. H. Martin, P. C. Wainwright, B. Masschaele, and M. Dierick. 2018. Building trophic specializations that result in substantial niche partitioning within a young adaptive radiation. J. Anat. 232:173-185.

Hopkins, C. D. 1995. Convergent designs for electrogenesis and electroreception. Curr. Opin. Neurobiol. 5:769-777.

James, M. E., M. J. Wilkinson, H. L. North, J. Engelstädter, and D. Ortiz-Barrientos. 2020. A framework to quantify phenotypic and genotypic parallel evolution. bioRxiv, doi: 10.1101/2020.02.05.936450.

Jones, F. C., M. G. Grabherr, Y. F. Chan, P. Russell, E. Mauceli, J. Johnson, R. Swofford, M. Pirun, M. C. Zody, S. White, E. Birney, S. Searle, J. Schmutz, J. Grimwood, M. C. Dickson, R. M. Myers, C. T. Miller, B. R. Summers, A. K. Knecht, S. D. Brady, H. Zhang, A. A. Pollen, T. Howes, C. Amemiya, J. Baldwin, T. Bloom, D. B. Jaffe, R. Nicol, J. Wilkinson, E. S. Lander, F. Di Palma, K. Lindblad-Toh, and D. M. Kingsley. 2012. The genomic basis of adaptive evolution in threespine sticklebacks. Nature 484:55-61.

Jost, M. C., D. M. Hillis, Y. Lu, J. W. Kyle, H. A. Fozzard, and H. H. Zakon. 2008. Toxinresistant sodium channels: Parallel adaptive evolution across a complete gene family. Mol. 
Biol. Evol. 25:1016-1024. Oxford Academic.

Juenger, T. E., S. Sen, K. A. Stowe, and E. L. Simms. 2005. Epistasis and genotype-environment interaction for quantitative trait loci affecting flowering time in Arabidopsis thaliana. Pp. 87-105 in Genetica.

Katz, P. S. 2006. Comparative Neurophysiology: An Electric Convergence in Fish.

Kowalko, J. E., N. Rohner, T. A. Linden, S. B. Rompani, W. C. Warren, R. Borowsky, C. J. Tabin, W. R. Jeffery, and M. Yoshizawa. 2013. Convergence in feeding posture occurs through different genetic loci in independently evolved cave populations of Astyanax mexicanus. Proc. Natl. Acad. Sci. U. S. A. 110:16933-16938.

Lander, E., and L. Kruglyak. 1995. Genetic dissection of complex traits: Guidelines for interpreting and reporting linkage results. Nat. Genet. 11:241-247.

Lander, E. S., and S. Botstein. 1989. Mapping mendelian factors underlying quantitative traits using RFLP linkage maps. Genetics 121:185.

Linnen, C. R., Y. P. Poh, B. K. Peterson, R. D. H. Barrett, J. G. Larson, J. D. Jensen, and H. E. Hoekstra. 2013. Adaptive evolution of multiple traits through multiple mutations at a single gene. Science (80-. ). 339:1312-1316. American Association for the Advancement of Science.

Liu, J., M. Aoki, I. Illa, C. Wu, M. Fardeau, C. Angelini, C. Serrano, J. Andoni Urtizberea, F. Hentati, M. Ben Hamida, S. Bohlega, E. J. Culper, A. A. Amato, K. Bossie, J. Oeltjen, K. Bejaoui, D. McKenna-Yasek, B. A. Hosler, E. Schurr, K. Arahata, P. J. De Jong, and R. H. Brown. 1998. Dysferlin, a novel skeletal muscle gene, is mutated in Miyoshi myopathy and limb girdle muscular dystrophy. Nat. Genet. 20:31-36.

Liu, R. K., and A. A. Echelle. 2013. Behavior of the Catarina pupfish (cyprinodontidae: Megupsilon aporus), a severely imperiled species. Southwest. Nat. 58:1-7. Southwestern Association of Naturalists.

Losos, J. B. 2009. Lizards in an Evolutionary Tree: Ecology and Adaptive Radiation of Anoles. University of California Press, Berkeley.

Lynch, M., and B. Walsh. 1998. Genetics and Analysis of Quantitative Traits. Vol. 1. Sinauer Associates, Sunderland, MA, Sunderland, MA.

Marsden, C. D., Y. Lee, K. Kreppel, A. Weakley, A. Cornel, H. M. Ferguson, E. Eskin, and G. C. Lanzaro. 2014. Diversity, differentiation, and linkage disequilibrium: Prospects for association mapping in the malaria vector anopheles arabiensis. G3 Genes, Genomes, Genet. 4:121-131. Genetics Society of America.

Martin, C. H., J. E. Crawford, B. J. Turner, and L. H. Simons. 2016. Diabolical survival in Death Valley: recent pupfish colonization, gene flow and genetic assimilation in the smallest species range on earth. Proc. R. Soc. B Biol. Sci. 283:20152334.

Martin, C. H., J. S. Cutler, J. P. Friel, C. Dening Touokong, G. Coop, and P. C. Wainwright. 2015. Complex histories of repeated gene flow in Cameroon crater lake cichlids cast doubt on one of the clearest examples of sympatric speciation. Evolution (N. Y). 69:1406-1422.

Martin, C. H., P. A. Erickson, and C. T. Miller. 2017. The genetic architecture of novel trophic specialists: larger effect sizes are associated with exceptional oral jaw diversification in a pupfish adaptive radiation. Mol. Ecol. 26:624-638.

Martin, C. H., and L. C. Feinstein. 2014. Novel trophic niches drive variable progress towards ecological speciation within an adaptive radiation of pupfishes. Mol. Ecol. 23:1846-1862.

Martin, C. H., and P. C. Wainwright. 2013. A Remarkable Species Flock of Cyprinodon Pupfishes Endemic to San Salvador Island, Bahamas. Bull. Peabody Museum Nat. Hist. 
54:231-241.

McGirr, J. A., and C. H. Martin. 2020. Ecological divergence in sympatry causes gene misexpression in hybrids. Mol. Ecol. 29:2707-2721.

McGirr, J. A., and C. H. Martin. 2021. Few Fixed Variants between Trophic Specialist Pupfish Species Reveal Candidate Cis-Regulatory Alleles Underlying Rapid Craniofacial Divergence. Mol. Biol. Evol. 38:405-423.

McGirr, J. A., and C. H. Martin. 2016. Novel candidate genes underlying extreme trophic specialization in Caribbean pupfishes. Mol. Biol. Evol. 34:msw286. Narnia.

Morjan, C. L., and L. H. Rieseberg. 2004. How species evolve collectively: implications of gene flow and selection for the spread of advantageous alleles. Mol. Ecol. 13:1341-1356. John Wiley \& Sons, Ltd.

Nelson, T. C., and W. A. Cresko. 2018. Ancient genomic variation underlies repeated ecological adaptation in young stickleback populations. Evol. Lett. 2:9-21.

Nyholt, D. R. 2000. All LODs are not created equal. Am. J. Hum. Genet. 67:282-288. University of Chicago Press.

O’brown, N. M., B. R. Summers, F. C. Jones, S. D. Brady, and D. M. Kingsley. 2015. A recurrent regulatory change underlying altered expression and Wnt response of the stickleback armor plates gene EDA. Elife 2015.

Orr, H. A. 2006. The distribution of fitness effects among beneficial mutations in Fisher's geometric model of adaptation. J. Theor. Biol. 238:279-285.

Paaby, A. B., and M. V. Rockman. 2013. The many faces of pleiotropy. Elsevier Current Trends.

Parsons, T. E., S. M. Weinberg, K. Khaksarfard, R. N. Howie, M. Elsalanty, J. C. Yu, and J. J. Cray. 2014. Craniofacial shape variation in Twist1+/- mutant mice. Anat. Rec. 297:826833. Blackwell Publishing Inc.

Pavlidis, P., D. Živković, A. Stamatakis, and N. Alachiotis. 2013. SweeD: Likelihood-based detection of selective sweeps in thousands of genomes. Mol. Biol. Evol. 30:2224-2234. Mol Biol Evol.

Richards, E. J., and C. H. Martin. 2017. Adaptive introgression from distant Caribbean islands contributed to the diversification of a microendemic adaptive radiation of trophic specialist pupfishes. PLoS Genet. 13:e1006919.

Richards, E. J., J. A. McGirr, J. R. Wang, M. E. St. John, J. W. Poelstra, M. J. Solano, D. C. O'Connell, B. J. Turner, and C. H. Martin. 2021. A vertebrate adaptive radiation is assembled from an ancient and disjunct spatiotemporal landscape. Proc. Natl. Acad. Sci. 118:e2011811118. Proceedings of the National Academy of Sciences.

Risch, N. 1990. Linkage strategies for genetically complex traits. I. Multilocus models. Am. J. Hum. Genet. 46:222-228.

Rosenblum, E. B., C. E. Parent, and E. E. Brandt. 2014. The molecular basis of phenotypic convergence. Annu. Rev. Ecol. Evol. Syst. 45:203-226.

Russello, M. A., M. D. Waterhouse, P. D. Etter, and E. A. Johnson. 2015. From promise to practice: Pairing non-invasive sampling with genomics in conservation. PeerJ 2015:e1106. PeerJ Inc.

Schluter, D., E. A. Clifford, M. Nemethy, and J. S. McKinnon. 2004. Parallel evolution and inheritance of quantitative traits. Am. Nat. 163:809-822. The University of Chicago Press.

Sen, Ś., J. M. Satagopan, K. W. Broman, and G. A. Churchill. 2007. R/qtlDesign: Inbred line cross experimental design. Mamm. Genome 18:87-93.

Shirley, M. D., H. Tang, C. J. Gallione, J. D. Baugher, L. P. Frelin, B. Cohen, P. E. North, D. A. 
Marchuk, A. M. Comi, and J. Pevsner. 2013. Sturge-Weber Syndrome and Port-Wine Stains Caused by Somatic Mutation in GNAQ . N. Engl. J. Med. 368:1971-1979. New England Journal of Medicine (NEJM/MMS).

St. John, M. E., K. Dixon, and C. H. Martin. 2020a. Oral shelling within an adaptive radiation of pupfishes: testing the adaptive function of novel nasal protrusion and behavioral preference. J. Fish Biol. 1-9.

St. John, M. E., R. Holzman, and C. H. Martin. 2020b. Rapid adaptive evolution of scale-eating kinematics to a novel ecological niche. J. Exp. Biol. jeb.217570.

Stern, D. L. 2013. The genetic causes of convergent evolution. Nat. Rev. Genet. 14:751-764. Nature Publishing Group.

Stern, D. L., and V. Orgogozo. 2008. The loci of evolution: How predictable is genetic evolution?

Stevenson, M. M. 1981. Karyomorphology of Several Species of Cyprinodon. Copeia 1981:494. JSTOR.

Stuart, Y. E., T. Veen, J. N. Weber, D. Hanson, M. Ravinet, B. K. Lohman, C. J. Thompson, T. Tasneem, A. Doggett, R. Izen, N. Ahmed, R. D. H. Barrett, A. P. Hendry, C. L. Peichel, and D. I. Bolnick. 2017. Contrasting effects of environment and genetics generate a continuum of parallel evolution. Nat. Ecol. Evol. 1:158. Nature Publishing Group.

Taylor, J., and D. Butler. 2017. R package ASMap: Efficient genetic linkage map construction and diagnosis. J. Stat. Softw., doi: 10.18637/jss.v079.i06.

Taylor, R. S., M. Manseau, R. L. Horn, S. Keobouasone, G. B. Golding, and P. J. Wilson. 2020. The role of introgression and ecotypic parallelism in delineating intraspecific conservation units. Mol. Ecol. 29:2793-2809. Blackwell Publishing Ltd.

Teng, C. S., M. C. Ting, D. T. Farmer, M. Brockop, R. E. Maxson, and J. G. Crump. 2018. Altered bone growth dynamics prefigure craniosynostosis in a zebrafish model of SaethreChotzen syndrome. Elife 7. eLife Sciences Publications Ltd.

Thompson, K. A., M. M. Osmond, and D. Schluter. 2019. Parallel genetic evolution and speciation from standing variation. Evol. Lett. 3:129-141. Wiley.

Wagner, G. P., J. P. Kenney-Hunt, M. Pavlicev, J. R. Peck, D. Waxman, and J. M. Cheverud. 2008. Pleiotropic scaling of gene effects and the "cost of complexity." Nature 452:470-472. Nature Publishing Group.

Wittkopp, P. J., B. L. Williams, J. E. Selegue, and S. B. Carroll. 2003. Drosophila pigmentation evolution: Divergent genotypes underlying convergent phenotypes. Proc. Natl. Acad. Sci. U. S. A. 100:1808-1813. National Academy of Sciences.

Xie, K. T., G. Wang, A. C. Thompson, J. I. Wucherpfennig, T. E. Reimchen, A. D. C. MacColl, D. Schluter, M. A. Bell, K. M. Vasquez, and D. M. Kingsley. 2019. DNA fragility in the parallel evolution of pelvic reduction in stickleback fish. Science (80-. ). 363:81-84.

Yang, J., N. A. Zaitlen, M. E. Goddard, P. M. Visscher, and A. L. Price. 2014. Advantages and pitfalls in the application of mixed-model association methods.

Yu, J., G. Pressoir, W. H. Briggs, I. V. Bi, M. Yamasaki, J. F. Doebley, M. D. McMullen, B. S. Gaut, D. M. Nielsen, J. B. Holland, S. Kresovich, and E. S. Buckler. 2006. A unified mixedmodel method for association mapping that accounts for multiple levels of relatedness. Nat. Genet. 38:203-208. Nature Publishing Group. 


\section{Tables and Figures}

Table 1. Maximum LOD scores for all 29 traits measured in Little Lake and Crescent Pond mapping crosses. A genome scan with a single-QTL model by Haley-Knott regression was used to identify the position with the highest LOD score, 95\% Bayesian credible intervals, and the genome-wide significance level for each trait $\left(P<0.1: \bullet ; P<0.05:{ }^{*}\right)$. We also report the scaffold numbers of genomic regions that fell within the $95 \%$ credible intervals associated with the maximum LOD position for each trait, the number of individuals phenotyped, percent variance explained (PVE) by the max LOD region, and the uncorrected $P$-value associated with each max LOD region.

\begin{tabular}{|c|c|c|c|c|c|c|c|}
\hline Trait & Population & Scaffold & $\begin{array}{l}\text { Max } \\
\text { LOD }\end{array}$ & $\begin{array}{c}\text { Genome - } \\
\text { wide } \\
\text { Significance }\end{array}$ & n & PVE & $\begin{array}{l}x^{2} p- \\
\text { Value }\end{array}$ \\
\hline \multirow{2}{*}{$\begin{array}{l}\text { Lower Jaw } \\
\text { Length }\end{array}$} & $\begin{array}{l}\text { Crescent } \\
\text { Pond }\end{array}$ & $\begin{array}{c}53,7087,2336 \\
6275,26,7335\end{array}$ & 2.89 & & 205 & 6.29 & 0.0013 \\
\hline & Little Lake & $24,4028,58,16$ & 3.30 & & 228 & 6.45 & 0.0005 \\
\hline \multirow{3}{*}{$\begin{array}{l}\text { Jaw closing } \\
\text { In-Lever }\end{array}$} & $\begin{array}{l}\text { Crescent } \\
\text { Pond }\end{array}$ & $31,4,451,19$ & 3.60 & & 204 & 7.81 & 0.0002 \\
\hline & Little Lake & & & • & & & \\
\hline & & $8,9588,8020$ & 4.11 & & 227 & 7.99 & 0.0001 \\
\hline \multirow{2}{*}{$\begin{array}{l}\text { Jaw Opening } \\
\text { In-Lever }\end{array}$} & $\begin{array}{l}\text { Crescent } \\
\text { Pond }\end{array}$ & 6086,11 & 2.43 & & 205 & 5.32 & 0.0037 \\
\hline & Little Lake & 43 & 2.98 & & 227 & 5.87 & 0.0010 \\
\hline \multirow{2}{*}{$\begin{array}{l}\text { Palatine } \\
\text { Height }\end{array}$} & $\begin{array}{l}\text { Crescent } \\
\text { Pond }\end{array}$ & $34,22,6304$ & 2.90 & & 205 & 6.31 & 0.0013 \\
\hline & Little Lake & 11 & 2.73 & & 228 & 5.36 & 0.0019 \\
\hline \multirow{2}{*}{$\begin{array}{l}\text { Suspensorium } \\
\text { Length }\end{array}$} & $\begin{array}{l}\text { Crescent } \\
\text { Pond }\end{array}$ & $\begin{array}{c}46,37,31,26,60 \\
7556,10198,22\end{array}$ & 3.54 & & 204 & 7.68 & 0.0003 \\
\hline & Little Lake & 11 & 3.51 & & 227 & 6.88 & 0.0003 \\
\hline \multirow{3}{*}{$\begin{array}{l}\text { Dentigerous } \\
\text { Arm Width }\end{array}$} & $\begin{array}{l}\text { Crescent } \\
\text { Pond }\end{array}$ & $52,13137,40$ & 2.19 & & 202 & 4.87 & 0.0065 \\
\hline & Little Lake & & & . * & & & \\
\hline & & $24,4028,58,16$ & 4.05 & & 228 & 7.85 & 0.0001 \\
\hline \multirow{2}{*}{$\begin{array}{l}\text { Maxilla } \\
\text { Length }\end{array}$} & $\begin{array}{l}\text { Crescent } \\
\text { Pond }\end{array}$ & $\begin{array}{c}27,593,4,31 \\
451,19\end{array}$ & 2.67 & & 204 & 5.85 & 0.0021 \\
\hline & Little Lake & 56 & 3.03 & & 228 & 5.94 & 0.0009 \\
\hline \multirow{2}{*}{$\begin{array}{l}\text { Dentigerous } \\
\text { Arm Base }\end{array}$} & $\begin{array}{l}\text { Crescent } \\
\text { Pond }\end{array}$ & $\begin{array}{c}27,593,4,31 \\
451,19\end{array}$ & 2.98 & & 205 & 6.47 & 0.0011 \\
\hline & Little Lake & 26 & 3.70 & & 228 & 7.21 & 0.0002 \\
\hline
\end{tabular}




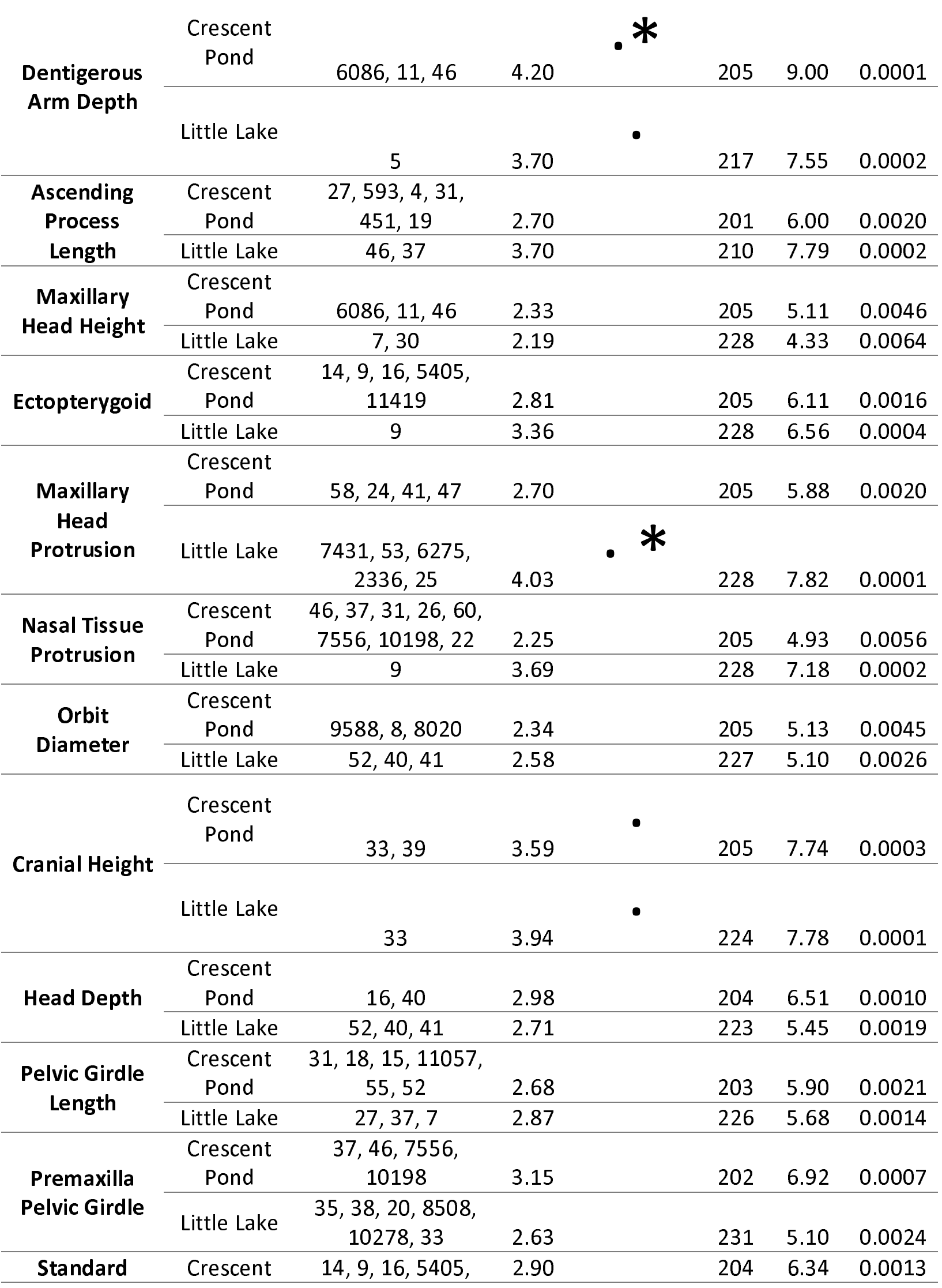




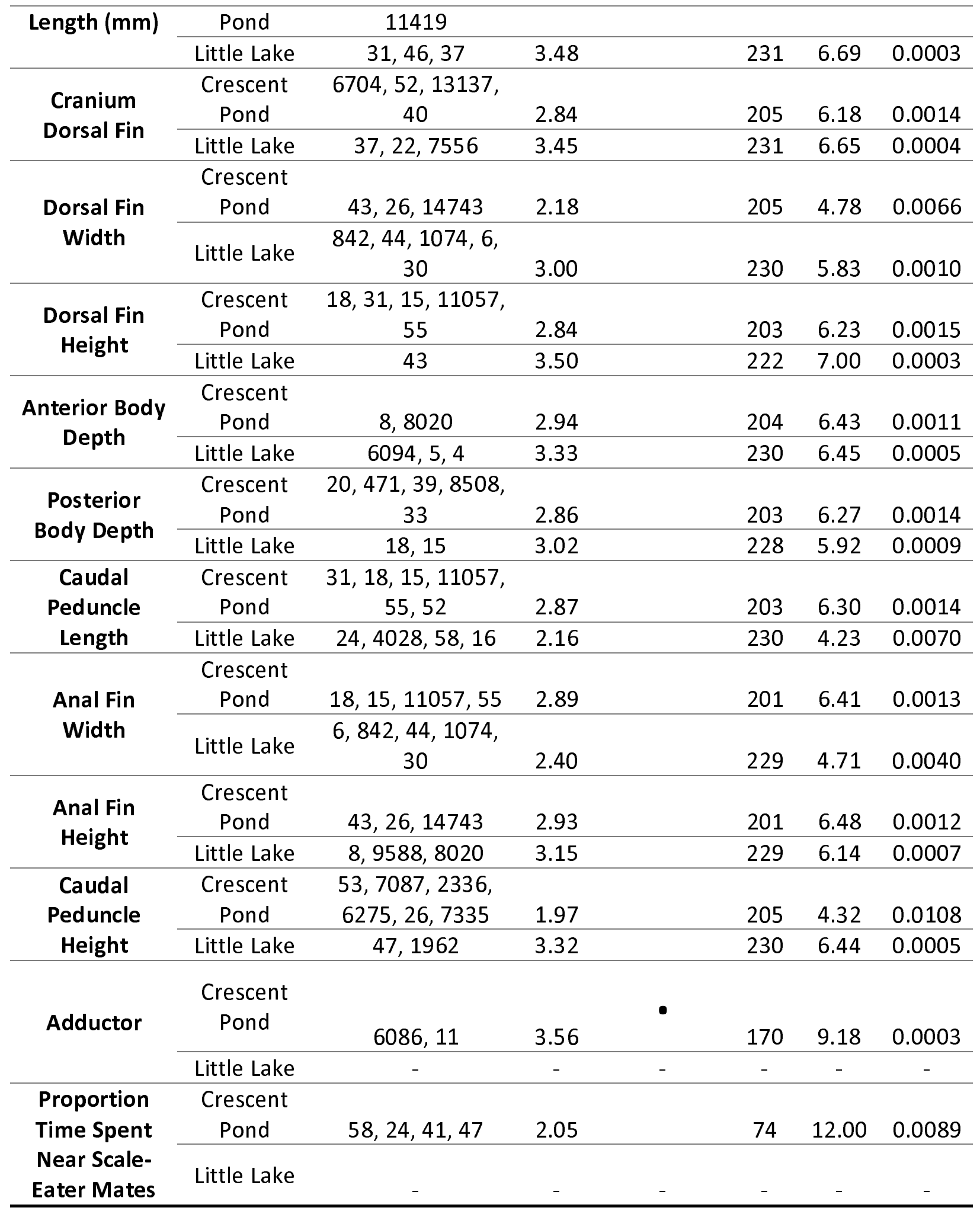


Table 2. Position of maximum LOD score and 95\% credible intervals for each trait in the Little Lake linkage map and the Crescent Pond linkage map. Colors represent corresponding linkage groups across lakes. Asterisks represent traits that were marginally significant at the $P<0.1$ level in the genome scan.

\begin{tabular}{|c|c|c|c|c|c|c|c|c|}
\hline \multicolumn{4}{|l|}{ Little Lake } & \multicolumn{5}{|c|}{ Crescent Pond } \\
\hline \multirow[b]{2}{*}{ Trait } & \multicolumn{3}{|c|}{$\begin{array}{c}\text { Position } \\
\text { genomewide }\end{array}$} & \multirow[b]{2}{*}{ Trait } & \multicolumn{4}{|c|}{$\begin{array}{c}\text { Position } \\
\text { genom ewide }\end{array}$} \\
\hline & Sig. LG & Max LOD score & $95 \% \mathrm{Cl}$ & & Sig. & LG & Max LOD score & $95 \% \mathrm{Cl}$ \\
\hline Cranial Height & * 1 & 259 & $(250,270)$ & Suspensorium Length & & 1 & 566 & $(20,730)$ \\
\hline Premaxilla to Pelvic Girdle & 1 & 146 & $(0,350)$ & Nasal Tissue Protrusion & & 1 & 570 & $(0,740)$ \\
\hline Cranium to Dorsal Fin & 2 & 303 & $(160,380)$ & Premaxilla to Pelvic Girdle & & 1 & 568 & $(310,600)$ \\
\hline Lower Jaw Length & 3 & 9 & $(0,340)$ & Ectopterygoid & & 3 & 272 & $(0,560)$ \\
\hline Dentigerous Arm Width & 3 & 9 & $(0,340)$ & Standard Length $(\mathrm{mm})$ & & 3 & 50 & $(40,500)$ \\
\hline Caudal Peduncle Length & 3 & 168 & $(0,340)$ & Dentigerous Arm Width & & 4 & 317 & $(40,510)$ \\
\hline Dorsal Find width & 4 & 187 & $(10,310)$ & Cranium to Dorsal Fin & & 4 & 89 & $(30,510)$ \\
\hline Anal Fin Width & 4 & 14 & $(0,280)$ & Lower Jaw Length & & 5 & 136 & $(0,470)$ \\
\hline Dentigerous Arm Depth & 6 & 79 & $(20,90)$ & Caudal Peduncle height & & 5 & 381 & $(0,470)$ \\
\hline Anterior Body Depth & 6 & 289 & $(0,300)$ & Jaw Closing In-Lever & & 6 & 380 & $(150,410)$ \\
\hline Orbit Diameter & 8 & 266 & $(0,290)$ & Maxilla Length & & 6 & 468 & $(0,480)$ \\
\hline Head Depth & 8 & 206 & $(0,290)$ & Dentigerous Arm Base & & 6 & 107 & $(0,480)$ \\
\hline Jaw Closing In-Lever & 9 & 54 & $(40,90)$ & Ascending Proces Length & & 6 & 106 & $(0,470)$ \\
\hline Anal Fin Height & 9 & 100 & $(70,240)$ & Pelvic Girdle Length & & 8 & 370 & $(20,435)$ \\
\hline Maxillary Head Protrusion & * 10 & 35 & $(0,260)$ & Dorsal Fin Height & & 8 & 91 & $(0,380)$ \\
\hline Ascending Process Length & 12 & 119 & $(90,150)$ & Caudal Peduncle Length & & 8 & 258 & $(30,425)$ \\
\hline Standard Length $(\mathrm{mm})$ & 12 & 200 & $(50,210)$ & Anal Fin Width & & 8 & 190 & $(110,400)$ \\
\hline Ectopterygoid & 13 & 170 & $(150,180)$ & Maxillary Head Protrusion & & 9 & 300 & $(0,350)$ \\
\hline Nasal Tissue Protrusion & 13 & 193 & $(20,200)$ & $\begin{array}{l}\text { Proportion Time Spent Near } \\
\text { Scale-Eater Males }\end{array}$ & & 9 & 166 & $(50,340)$ \\
\hline Palatine Height & 14 & 147 & $(110,210)$ & Cranial Height & * & 10 & 204 & $(130,340)$ \\
\hline Suspensorium Length & 14 & 153 & $(70,180)$ & Posterior Body Depth & & 10 & 270 & $(0,330)$ \\
\hline Jaw Opening In-Lever & 16 & 58 & $(40,140)$ & Palatine Height & & 11 & 70 & $(0,310)$ \\
\hline Dorsal Fin Height & 16 & 52 & $(40,60)$ & Head Depth & & 12 & 111 & $(100,280)$ \\
\hline Pelvic Girdle Length & 17 & 50 & $(10,160)$ & Opening In-Lever & & 13 & 10 & $(0,90)$ \\
\hline Maxillary Head Height & 18 & 122 & $(30,160)$ & Dentigerous Arm Depth & $*$ & 13 & 2 & $(0,250)$ \\
\hline Caudal Peduncle Height & 19 & 44 & $(20,90)$ & Maxillary Head Height & & 13 & 170 & $(0,280)$ \\
\hline Dentigerous Arm Base & 21 & 74 & $(0,100)$ & Adductor Mandibulae Mass & $*$ & 13 & 2 & $(0,70)$ \\
\hline Maxilla Length & 22 & 40 & $(20,50)$ & Dorsal Fin Width & & 14 & 305 & $(30,330)$ \\
\hline Posterior Body Depth & 24 & 30 & $(10,30)$ & Anal Fin Width & & 14 & 330 & $(280,330)$ \\
\hline & & & & Orbit Diameter & & 16 & 107 & $(0,190)$ \\
\hline & & & & Anterior Body Depth & & 16 & 170 & $(10,220)$ \\
\hline
\end{tabular}


Table 3. Number of adaptive alleles and any genes within $20 \mathrm{kbp}$ found in trait QTL with maximum LOD scores for both lakes. Adaptive alleles were categorized as either standing genetic variation (SGV), introgression (Intro.), or de novo mutations, and were estimated independently for snail-eaters and scale-eaters in a previous study (Richards et al. 2021). Asterisks represent traits that were significant at the $\mathrm{P}<0.1$ level in the genome-wide scan, while crosses show traits that corresponded to the same locations in the alternate lake.

\begin{tabular}{|c|c|c|c|c|c|c|}
\hline \multirow[t]{2}{*}{ Traits } & \multirow[t]{2}{*}{ Gene } & \multicolumn{2}{|c|}{ Snail-Eater } & \multicolumn{3}{|c|}{ Scale-Eater } \\
\hline & & SGV & Intro. & SGV & Intro. & de novo \\
\hline \multirow{4}{*}{ Cranial Height* } & bri3bp & - & 26 & 28 & - & - \\
\hline & gnaq & 9 & - & 9 & - & - \\
\hline & $w d r 31$ & 18 & 2 & 20 & - & - \\
\hline & $\begin{array}{r}\text { Unannotated } \\
\text { Regions } \\
\end{array}$ & 1 & - & 11 & - & - \\
\hline \multirow{3}{*}{$\begin{array}{l}\text { Dentigerous Arm Width* } \\
\text { Female mate preferencet } \\
\text { Maxillary Head Protrusiont }\end{array}$} & cyp26b1 & - & 8 & 8 & - & - \\
\hline & $d y s f$ & - & - & 1 & - & - \\
\hline & $\begin{array}{r}\text { Unannotated } \\
\text { Regions } \\
\end{array}$ & - & 67 & 216 & - & 1 \\
\hline \multirow[t]{9}{*}{ Dentigerous Arm Depth* } & $\begin{array}{r}\text { Unannotated } \\
\text { Regions } \\
\end{array}$ & - & - & 1 & - & - \\
\hline & $c 0 \times 6 b 1$ & 8 & - & 8 & - & - \\
\hline & сур21a2 & - & - & 2 & - & - \\
\hline & $e v a 1 b$ & - & - & 2 & - & - \\
\hline & fhod3 & - & - & 2 & - & - \\
\hline & galnt1 & - & - & - & 17 & - \\
\hline & glipr2 & - & - & 3 & - & - \\
\hline & $h d a c 9 b$ & - & - & - & 1 & - \\
\hline & $m a g$ & - & - & 2 & - & - \\
\hline \multirow{11}{*}{$\begin{array}{c}\text { Maxillary Head Protrusion* } \\
\text { Lower Jaw Length } † \\
\text { Caudal Peduncle Height } †\end{array}$} & $\operatorname{map} 7 d 1$ & 25 & - & 25 & - & - \\
\hline & $\min d y 3$ & - & - & 8 & - & - \\
\hline & nacad & - & - & 2 & - & - \\
\hline & $p \times n 1$ & - & - & 1 & - & - \\
\hline & rasip1 & 13 & - & 13 & - & - \\
\hline & $s / c 2 a 3$ & 15 & - & 15 & - & - \\
\hline & steap4 & - & - & - & 26 & - \\
\hline & tbrg4 & - & - & 2 & - & - \\
\hline & them 4 & - & - & 5 & - & - \\
\hline & tnc & - & - & 1 & - & - \\
\hline & twist1 & - & - & - & - & 1 \\
\hline
\end{tabular}




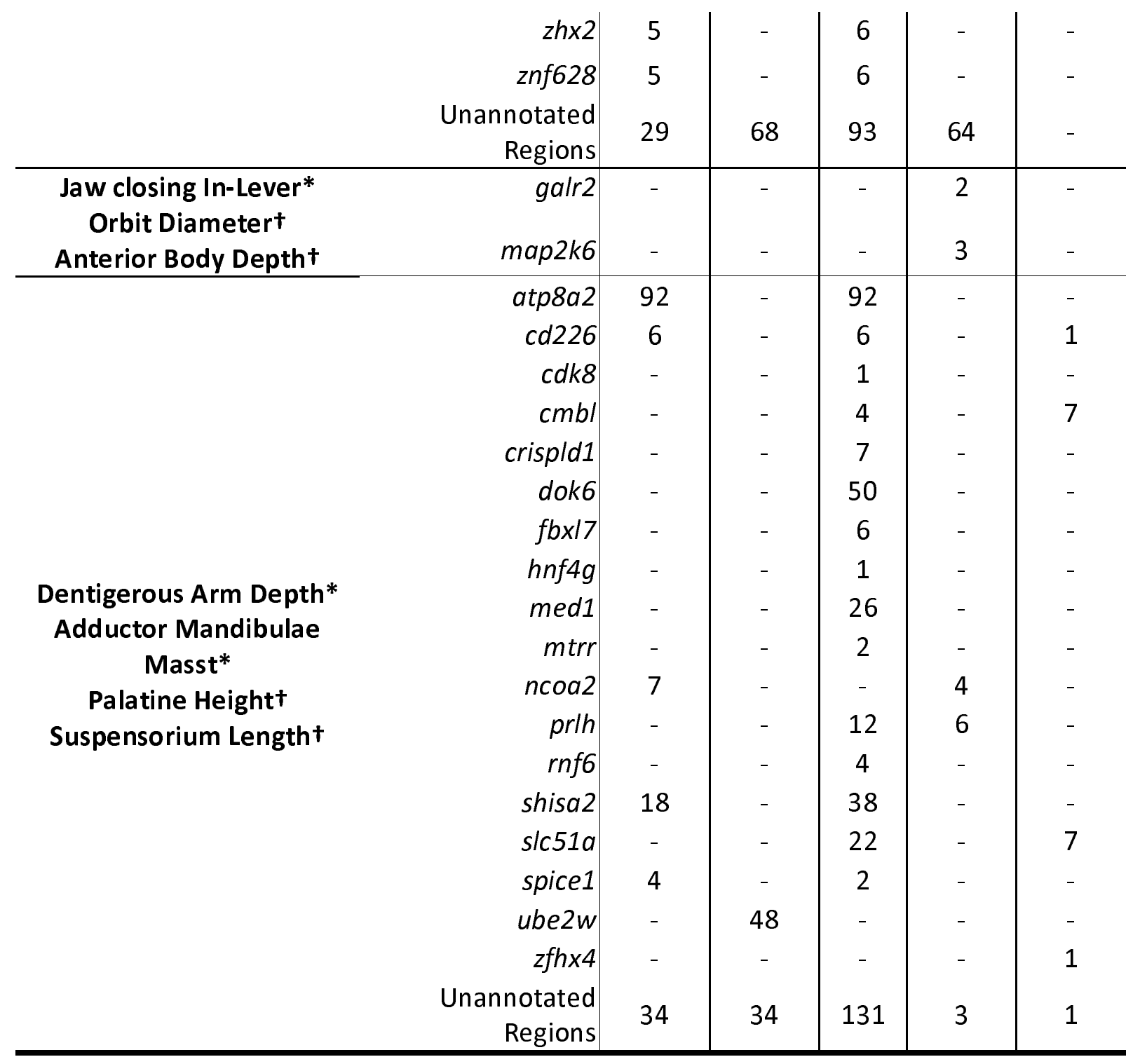


Figure 1 A) Representative photographs of F2 intercross cleared and double-stained specimen used for skeletal morphometrics. Points represent landmarks used to measure linear distances between skeletal traits. B) Table containing the two landmarks that correspond to each trait.

A)

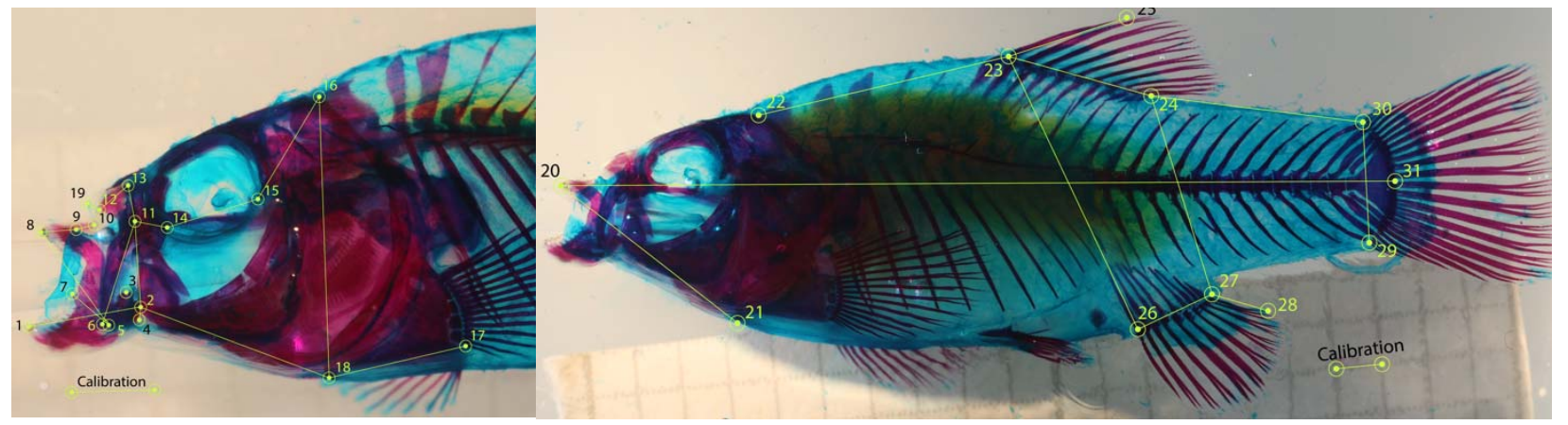

B)

\begin{tabular}{|c|c|c|c|c|c|}
\hline \multicolumn{3}{|l|}{ Head } & \multicolumn{3}{|l|}{ Body } \\
\hline Point 1 & Point 2 & Trait & Point 1 & Point 2 & Trait \\
\hline 1 & 2 & Lower Jaw Length & 20 & 21 & Premaxilla to Pelvic Girdle \\
\hline 2 & 3 & Jaw closing In-Lever & 20 & 31 & Standard Length \\
\hline 2 & 4 & Jaw Opening In-Lever & 22 & 23 & Cranium to Dorsal Fin \\
\hline 2 & 11 & Palatine Height & 23 & 24 & Dorsal Fin Width \\
\hline 2 & 18 & Suspensorium Length & 23 & 25 & Dorsal Fin Height \\
\hline 5 & 8 & Dentigerous Arm Width & 23 & 26 & Anterior Body Depth \\
\hline 6 & 11 & Maxilla Length & 24 & 27 & Posterior Body Depth \\
\hline 7 & 5 & Dentigerous Arm Base & 24 & 30 & Caudal Peduncle Length \\
\hline 8 & 9 & Dentigerous Arm Depth & 26 & 27 & Anal Fin Width \\
\hline 9 & 10 & Ascending Process Length & 27 & 28 & Anal Fin Height \\
\hline 11 & 13 & Maxillary Head Height & 29 & 30 & Caudal Peduncle Height \\
\hline 11 & 14 & Ectopterygoid & \multirow{7}{*}{\multicolumn{3}{|c|}{$\begin{array}{l}\text { For the Crescent Pond individuals, we } \\
\text { recorded sex and the mass of the adductor } \\
\text { mandibulae muscle before clearing and } \\
\text { staining each specimen. }\end{array}$}} \\
\hline 12 & 13 & Maxillary Head Protrusion & & & \\
\hline 12 & 19 & Nasal Tissue Protrusion & & & \\
\hline 14 & 15 & Orbit Diameter & & & \\
\hline 15 & 16 & Cranial Height & & & \\
\hline 16 & 18 & Head Depth & & & \\
\hline 17 & 18 & Pelvic Girdle Length & & & \\
\hline
\end{tabular}


bioRxiv preprint doi: https://doi.org/10.1101/2021.07.01.450661; this version posted July 22, 2021. The copyright holder for this preprint (which

was not certified by peer review) is the author/funder, who has granted bioRxiv a license to display the preprint in perpetuity. It is made available under aCC-BY 4.0 International license.

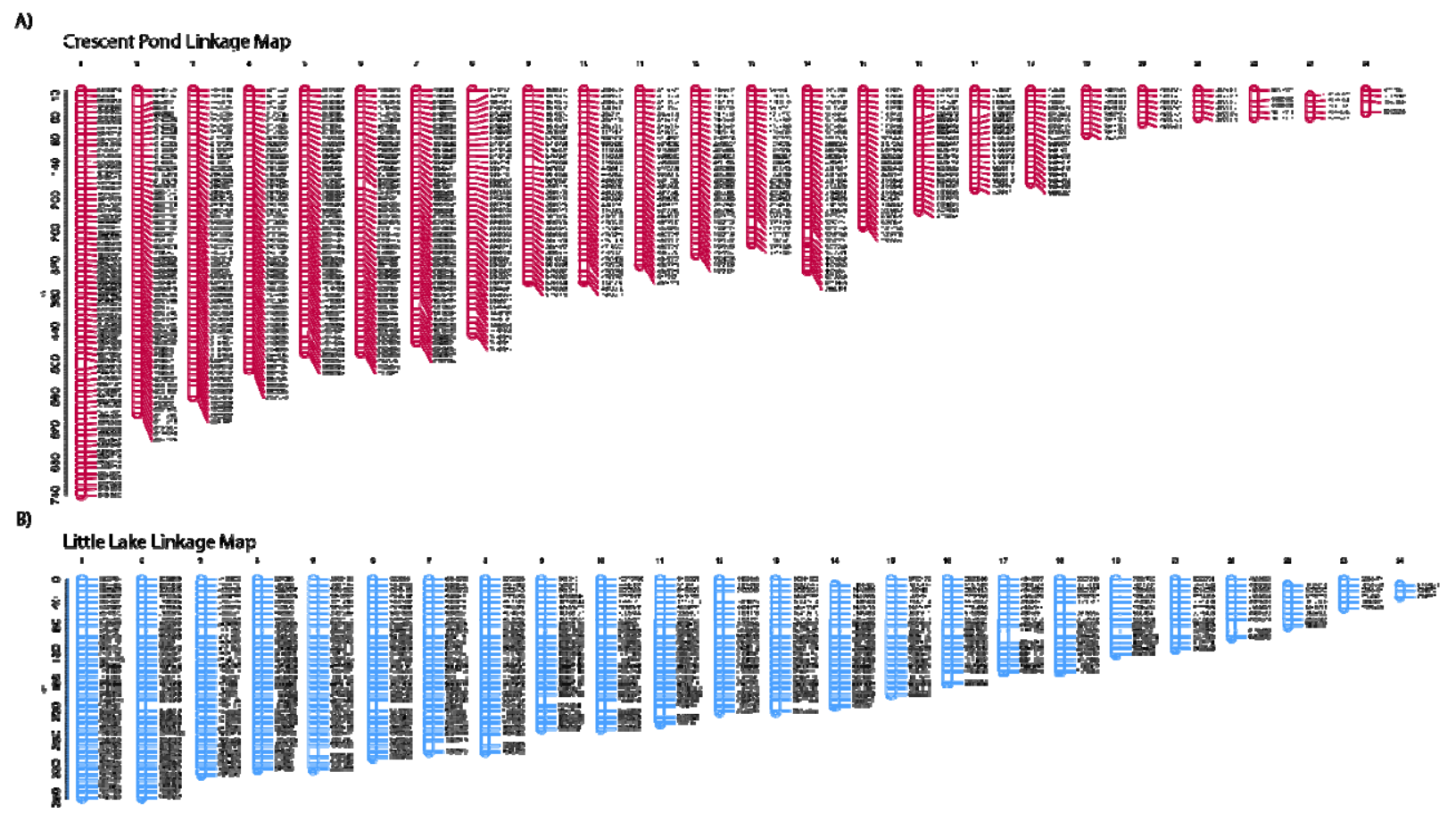

Figure 2 Linkage maps for A) Crescent Pond and B) Little Lake crosses. The Crescent Pond linkage map was estimated from 743 markers and the Little Lake linkage map was estimated from 540 markers. Both maps were generated from crosses between a scale-eater $(C$. desquamator) and snail-eater (C. brontotheroides) from the respective lakes. 


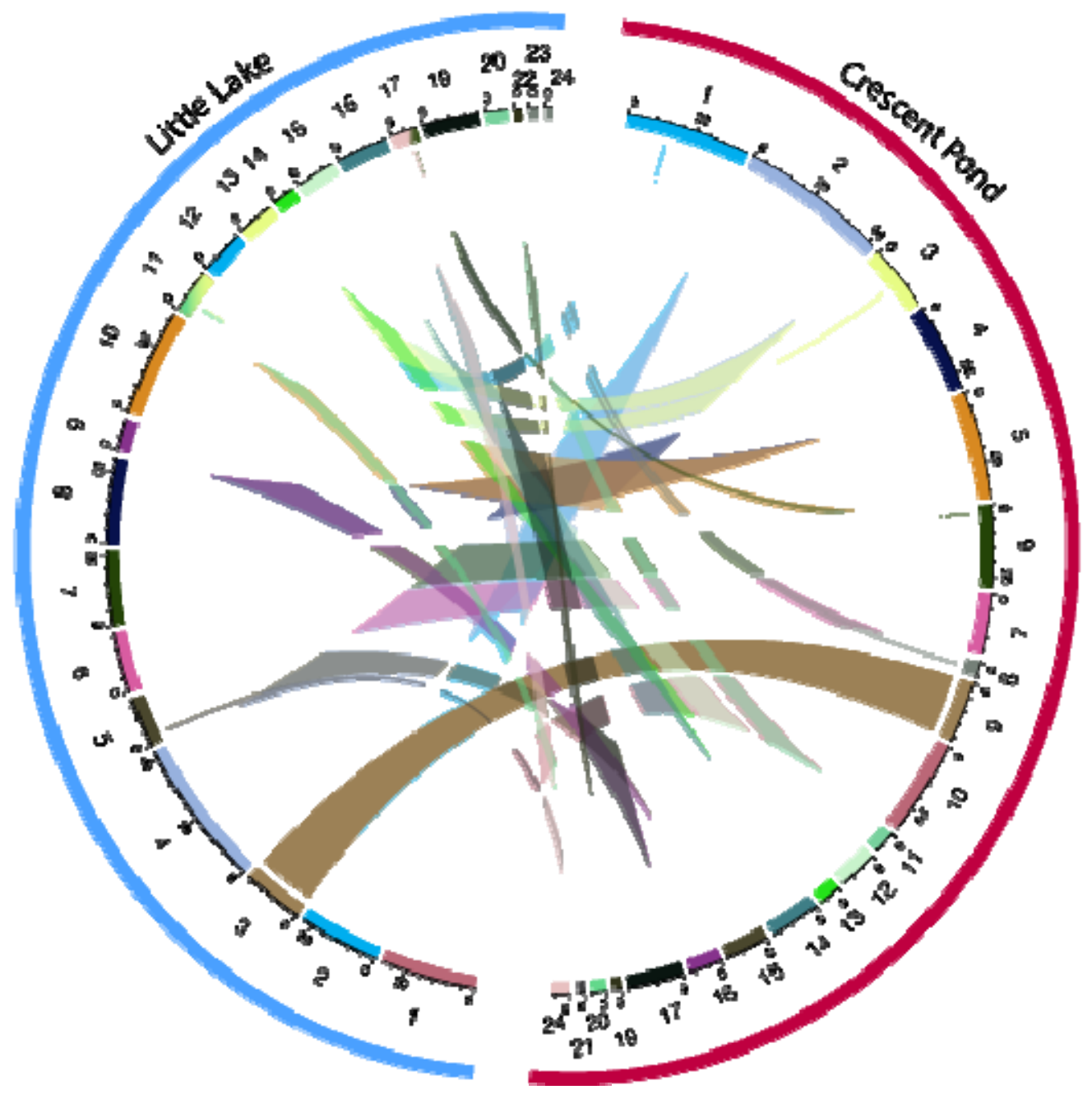

Figure 3 Circos plot depicting the relationship between the Crescent Pond (red) and Little Li linkage maps (blue), which share 324 markers within $10 \mathrm{kbp}$ of one another. Numbers surrounding each semi-circle represent linkage group numbers in each lake. Markers that are shared across lakes are connected via the colored lines. 


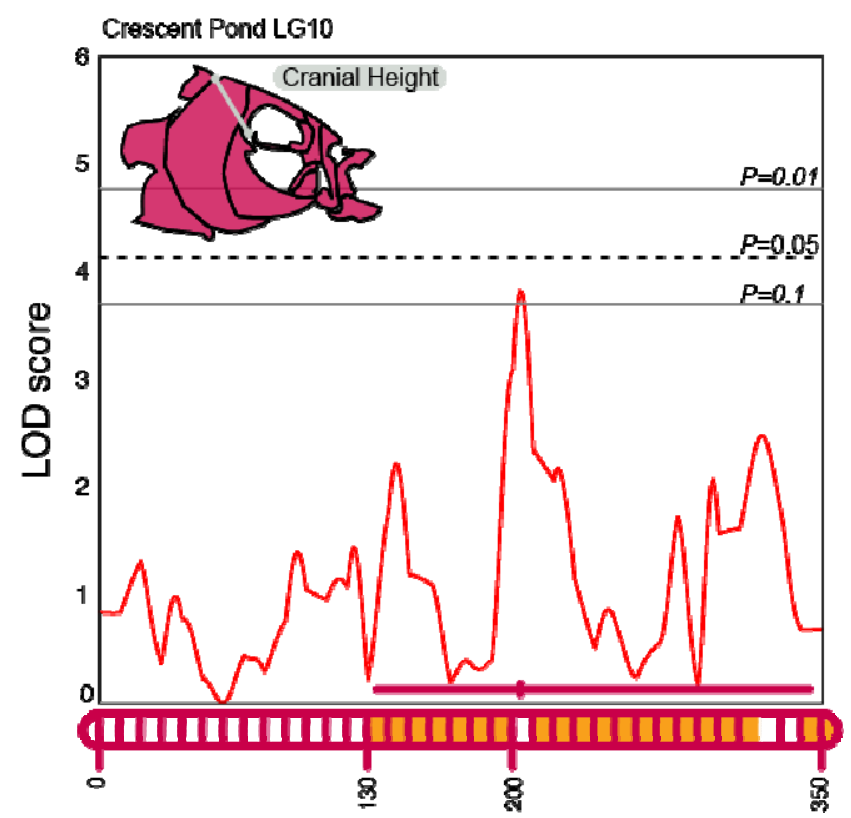

Figure 4 LOD

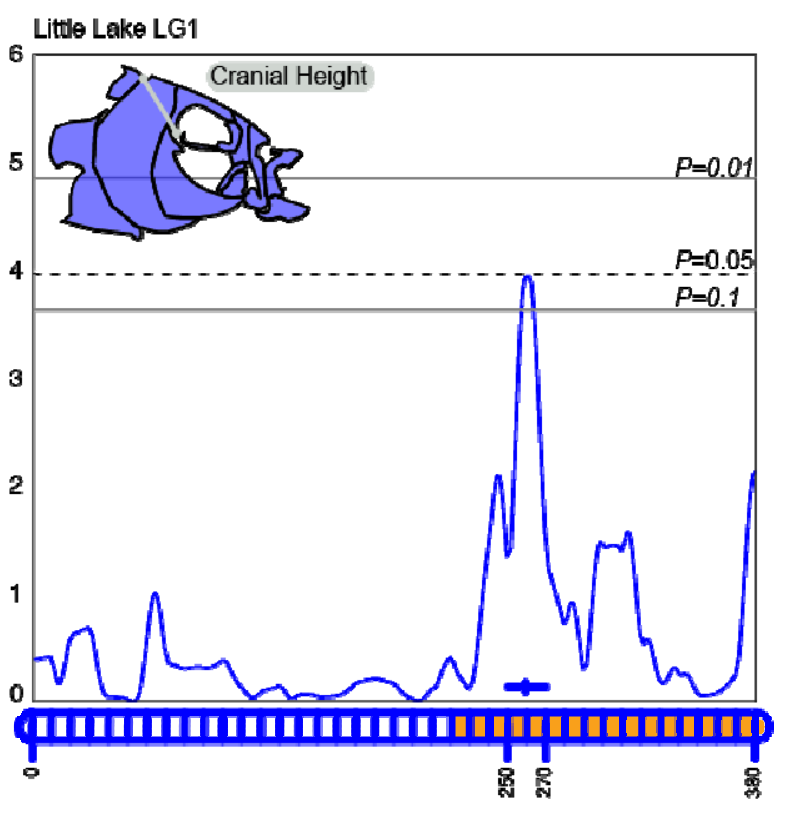

profile for cranial height in Crescent Pond

(red) and Little Lake (blue) F2 hybrids. LOD profiles were estimated by a Haley-Knott regression and are plotted relative to the position along the implicated linkage group (LG 10 for Crescent Pond, LG 1 for Little Lake) which are represented along the X-axis. Genome wide significance levels of $\mathrm{P}=0.1,0.05$, and 0.01 are shown by the grey horizontal lines. Linkage groups along the $\mathrm{X}$-axis also show the position of maximum LOD along with $95 \%$ Bayesian credible intervals. The orange fill color within the linkage groups corresponds to overlapping regions of scaffold 33 between crosses. 

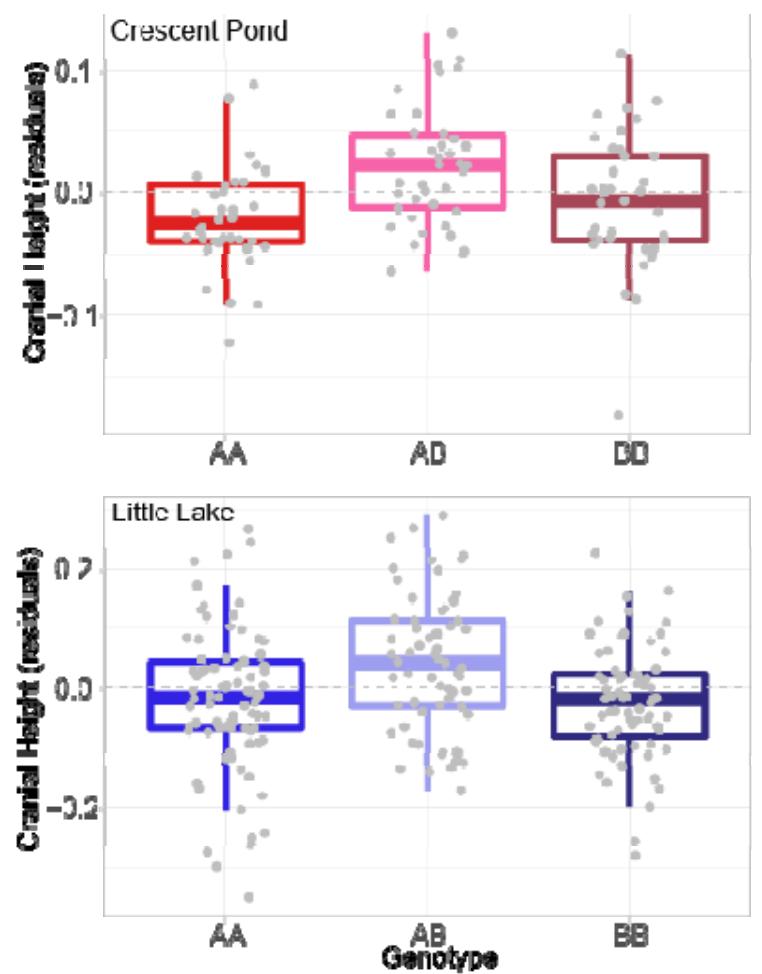

Figure 5 Cranial height phenotypes (size-corrected residuals) for each genotype in Crescent Pond (red) and Little Lake (blue). Both lakes show that heterozygotes (AB) exhibit greater cranial heights than homozygous parental genotypes. 